\title{
Flow-driven compaction of a fibrous porous medium
}

\author{
Daniel T. Paterson, ${ }^{1}$ Tom S. Eaves, ${ }^{2}$ Duncan R. Hewitt, ${ }^{3}$ Neil J. Balmforth, ${ }^{2}$ \\ and D. Mark Martinez ${ }^{1}$ \\ ${ }^{1}$ Department of Chemical and Biological Engineering, University of British Columbia, \\ Vancouver, BC, V6T 1Z4, Canada \\ ${ }^{2}$ Department of Mathematics, University of British Columbia, Vancouver, BC, V6T 1Z2, Canada \\ ${ }^{3}$ Department of Applied Mathematics and Theoretical Physics, University of Cambridge, \\ Cambridge, CB3 OWA, United Kingdom
}

(Received 23 August 2018; published 16 July 2019)

\begin{abstract}
A combined theoretical and experimental study is presented for the flow-induced compaction of a one-dimensional fibrous porous medium near its gel point for deformation at low and high rates. The theory is based on a two-phase model in which the permeability is a function of local solid fraction, and the deformation of the solid is resisted by both a compressive yield stress and a rate-dependent bulk viscosity. All three material properties are parameterized and calibrated for cellulose fibers using sedimentation, permeation, and filtration experiments. It is shown that the incorporation of rate-dependence in the solid stress significantly improves the agreement between theory and experiment when the drainage flow is relatively rapid. The model is extended to rates outside the range where it was calibrated to understand the dynamics of a standard test for pulp suspensions: the Canadian Standard Freeness test. The model adequately captures all of the experimental findings, including the score of the freeness test, which is found to be sensitively controlled by the bulk solid viscosity and to a lesser degree by the permeability law, but depends only weakly on the compressive yield stress.
\end{abstract}

DOI: 10.1103/PhysRevFluids.4.074306

\section{INTRODUCTION}

A suspension of fibers can establish a connected network that supports stress and resists deformation even at relatively low solid concentrations. This structure provides a porous matrix that can be substantially compacted and reorganized by flow, at solid volume fractions ranging from as low as a fraction of a percent, up to close to the maximum packing. The dynamics of such consolidation is relevant to a variety of problems in the physical and engineering sciences, including in the pulp and paper industry where the processing of suspensions of cellulose fibers constitutes an extensive and costly enterprise.

The purpose of the present paper is to explore the flow-induced compaction of a fibrous porous medium from near its gel point up to much higher solid fraction, in situations in which deformations occur at increasingly higher rates. We complement a theoretical approach based on a two-phase model with an experimental investigation using a particular suspension of cellulose fibers. By comparing the two while ramping up the deformation rates, our goal is to provide insight into the effective rheology of the fiber suspension, and thereby constrain the fluid-structure interaction occurring at the microscale.

Phenomenological study of the compaction of two-phase mixtures dates back more than a century (e.g., Coe and Clevenger [1] studied "slime-settling" in 1916), with independent theoretical developments following over the next 30 years in studies of industrial filtration [2,3] and soil mechanics $[4,5]$. The concepts behind these modeling approaches have been extended, adapted, 
or rediscovered in a wide range of contexts, from geological compaction to waste treatment [6-11]. While the formulations of these models take a number of forms, they typically have two key constitutive ingredients that parameterize the properties of the compressible medium: a solidfraction-dependent permeability and a compressive stress gauging the "strength" of the material [6].

Although in some situations (such as classical poroelasticity), the porous matrix provides an elastic solid stress and deformations are recoverable, many complex suspensions can exhibit a significant plastic stress, owing to rearrangements, entanglement and damage on the microscale. In contexts for which the material strength can be interpreted partly as a compressive yield stress, it is not clear whether the solid resistance contains only that rate-independent contribution. Indeed, the bulk matrix may deform viscously if the stress exceeds the compressive yield stress, as in the theory of dense suspensions of spheres [12] or geological compaction [13], and such rate-dependent stresses are generic in the framework of two-phase flow theory [14]. Despite this being noted by a number of authors (e.g., Ref. [15]), for colloidal suspensions it is common to adopt only the compressive yield stress for the solid and argue that any rate-dependence is negligible. Recent work has, however, shown that a viscous term is crucially important in rapid mechanical dewatering tests on cellulose fiber suspensions at relatively large solid fractions [16]. Similar conclusions have been drawn from experiments involving the deformation of hydrogel spheres suspended in water $[17,18]$ or during capillary imbibition of water in paper sheets [19].

For our theoretical approach, we therefore adopt an extension of conventional two-phase models of compaction that includes a rate-dependent solid stress via a bulk viscosity (Sec. II). To compare with this theory, we then perform a sequence of sedimentation and drainage experiments with cellulose fiber suspensions (Sec. III). At the very low rates of compaction encountered in sedimentation, the solid is expected to mostly provide a compressive yield stress. With the faster compactions of gravity-driven drainage, rate-dependent stresses may become more prominent, allowing us to gauge whether a solid viscosity is again needed to reproduce the dynamics, but this time at relatively low solid fraction.

The sequence of experiments begins with steady state tests (gravitational sedimentation and compaction under a steady background flow, Secs. V and IV, respectively) designed to calibrate the compressive yield strength and permeability near the gel point; we document how the newly networked porous structure gains in strength as the solid fraction increases, and make contact with existing theories for dilute fiber suspensions [20,21]. We then explore the unsteady dynamics of sedimentation, without or with flow-through, and more rapid compactions induced by drainage (Sec. VI). To directly infer the deformation of the solid in these tests, we use particle image velocimetry (PIV) to track the motion of marked tracers in the suspension through a transparent side wall.

Finally, we turn to a widely used, gravity-driven drainage test for pulp, Canadian Standard Freeness (TAPPI Standard T221). In this industrial standard, a simple funnel geometry is employed to divert a fraction of the water drained from a pulp sample into a collection chamber. The volume of the diverted water, or the "freeness score," is set by the rate at which water leaves the pulp sample, which is controlled by the degree to which the cellulose matrix compacts and impedes flow as it drains. Although the test is beguilingly simple, the precise relationship between the freeness score and the different material properties of the fibrous medium is not transparent. Moreover, the only existing models of freeness are semi-empirical and based on the average filter resistance of the compacting pulp [22-24]. We therefore apply our two-phase model to the freeness test (Sec. VII), to explore whether its predictions match the observed freeness score, to dissect the underlying dynamics and to identify the controlling material properties. A more general aim is to determine whether the freeness test provides a useful device to interrogate the behavior of a deformable porous media under relatively rapid and substantial compaction.

\section{TWO-PHASE MODEL FORMULATION}

The geometries of the three types of compaction problems that we investigate are sketched in Figs. 1(a)-1(c). The first is a fixed volume chamber through which water can be recirculated to 

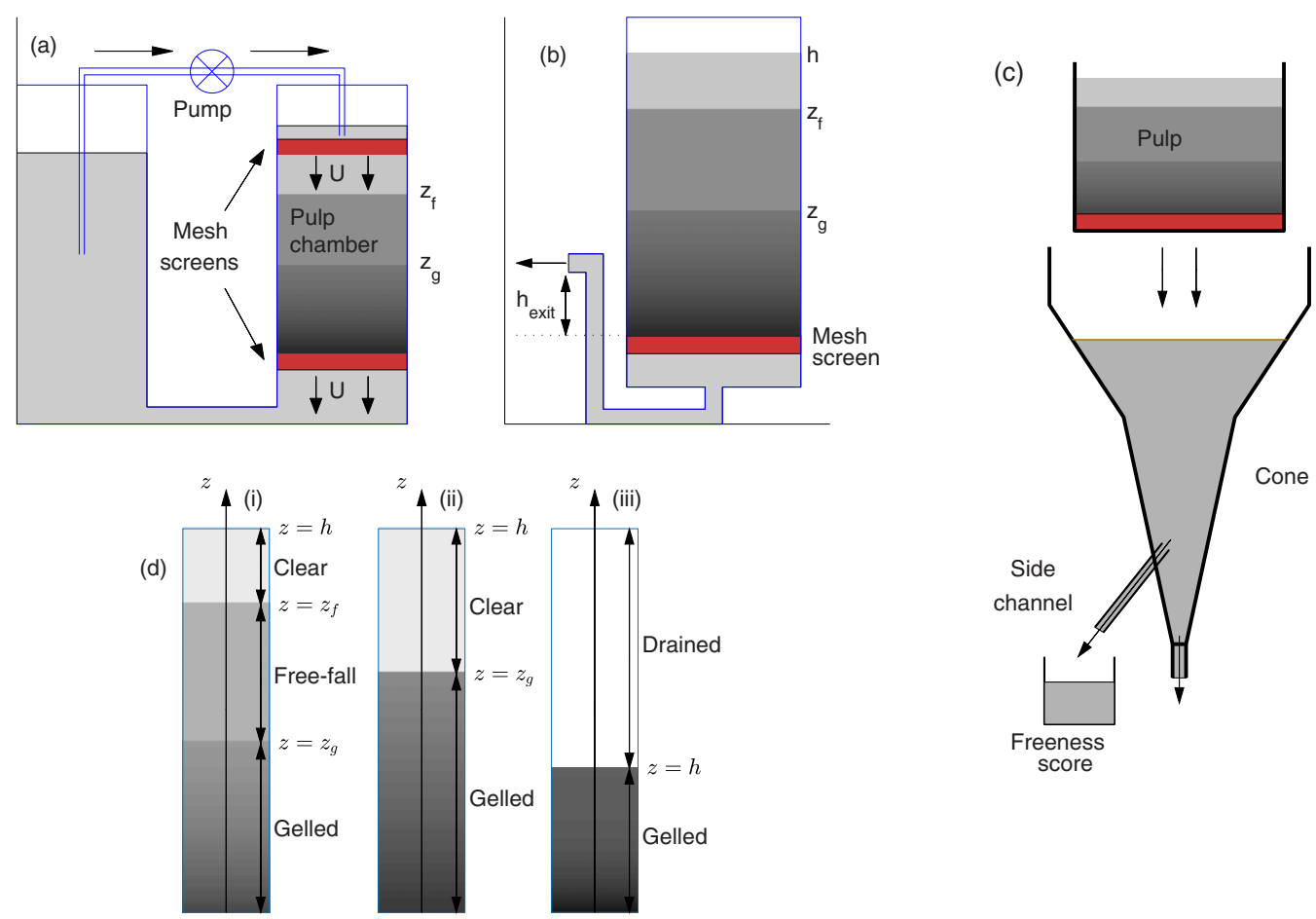

score

FIG. 1. Sketches of the flow configurations. The recirculation loop of the rectangular tank in the flowthrough experiments is sketched in panel (a), the tank for the drainage experiments in panel (b), and the freeness device in panel (c). Panel (d) illustrates the geometry of the three possible phase arrangements: (i) and (ii) illustrate the clear, freely falling and gelled layers in a closed container, in the manner of the sedimentation experiments; (iii) shows the final drainage stage of a configuration in which water is withdrawn and the top surface of the clear water layer meets that of the solid.

assist the gravitational sedimentation of fibers. In the second, water is allowed to drain through the base of a chamber containing the suspension so that the surface falls to a predetermined height. The third is the freeness test, where the water raining from the bottom of a sample of the suspension enters a funnel with a side channel. For all three, we ignore any interaction of the suspension with the side walls of the surrounding container and consider the vertical compaction of a one-dimensional two-phase medium with a non-Brownian solid, as discussed in Refs. [6-9]. In this formulation, spatial differentiation can lead to the three arrangements of fluid and solid sketched in Fig. 1(d).

\section{A. Governing equations}

The solid phase has volume fraction $\phi(z, t)$ and velocity $u(z, t)$, at height $z$ and time $t$. The solid and fluid densities, $\rho_{s}$ and $\rho_{f}$, are both assumed constant. The mixture is contained between a lower boundary at $z=0$ and upper surface at $z=h$ that is fixed for our models of fixed-volume compaction but falls over time in the drainage problems where water leaks through the bottom. At $t=0$, the mixture is assumed to be uniform, with initial solid fraction $\phi(z, 0)=\phi_{0}$. The solid phase cannot leave the column, and so

$$
\int_{0}^{h} \phi(z, t) d z=\phi_{0} h_{0}
$$

if $h_{0}$ denotes the initial height. 
Conservation of mass for the solid phase and the bulk medium implies

$$
\frac{\partial \phi}{\partial t}+\frac{\partial}{\partial z}(u \phi)=0 \quad \text { and } \quad \frac{\partial}{\partial z}\left[u \phi+u_{f}(1-\phi)\right]=0,
$$

where $u_{f}$ is the velocity of the fluid phase. In all of the variations of the problem that we consider, the medium is supported from below by a rigid surface that is permeable to the fluid, but not the solid. Thus, the relative velocity between phases is

$$
u_{f}-u=\frac{U(t)-u}{1-\phi},
$$

given that $U(t) \equiv \phi u+u_{f}(1-\phi)$ is the net bulk flow velocity, which represents the fluid leakage through the lower surface.

Adopting Terzaghi's principle [4], the total stress is composed of the pore pressure $p(z, t)$ and (compressive) effective solid stress $\mathcal{P}(z, t)$. With the neglect of inertia, the force balance on the bulk mixture demands that these stresses balance gravity; i.e.,

$$
\frac{\partial}{\partial z}(p+\mathcal{P})=-\rho g \equiv-\left[\rho_{f}+\phi\left(\rho_{s}-\rho_{f}\right)\right] g
$$

where $\rho$ is the bulk density and $g$ is gravity. Finally, Darcy's law provides the relation

$$
\frac{\partial p}{\partial z}+\rho_{f} g=\frac{\mu(1-\phi)}{k(\phi)}\left(u-u_{f}\right) \equiv \frac{\mu}{k(\phi)}(u-U),
$$

where $\mu$ is the fluid viscosity and $k(\phi)$ is the permeability of the solid matrix.

\section{Constitutive laws}

Inspired by the $\phi \ll 1$ asymptotic limit of an array of rigid rods (e.g., Ref. [21]), we define a permeability function,

$$
k(\phi)=\frac{A}{\phi} \log \left(\alpha \phi^{-1}\right) \quad(0<\phi \lesssim 0.006)
$$

where $A$ and $\alpha$ are parameters. Results presented by Higdon and Ford [21] suggest that this form is adequate over the required range of solid fraction. Away from this low- $\phi$ limit, we extend Eq. (6) by bridging to literature relationships [16], using a simple interpolation based on the variable $\log \phi$ (Appendix A 2 b).

If the solid fraction of the material lies below the network's gel fraction $\phi_{g}$, then we assume that the solid cannot sustain compressive stress and $\mathcal{P}=0$. If instead $\phi>\phi_{g}$, then we adopt a viscoplastic constitutive law (cf. Ref. [6]) in which the solid fails under compression and deforms provided the stress exceeds the compressive yield stress $P_{y}(\phi)$ :

$$
\mathcal{P}=-\left[\frac{P_{y}(\phi)}{\left|\frac{\partial u}{\partial z}\right|}+\Lambda(\phi)\right] \frac{\partial u}{\partial z}, \quad \text { if }|\mathcal{P}|>P_{y}(\phi),
$$

where the rate-dependence of the solid is modeled by a bulk extensional viscosity $\Lambda(\phi)$. When $|\mathcal{P}|<P_{y}(\phi)$, however, the solid is assumed to withstand the imposed stress without deforming and so $\partial u / \partial z=0$, with the solid stress remaining otherwise undetermined (as for any material with a yield condition, or a friction law with a threshold).

Near the gel point $\phi=\phi_{g}$, we adopt the simple power law (cf. Ref. [6])

$$
P_{y}(\phi)= \begin{cases}0, & \phi<\phi_{g}, \\ m\left(\phi-\phi_{g}\right)^{n}, & \phi>\phi_{g},\end{cases}
$$

with the experiments outlined in Sec. IV designed to determine the exponent $n$ and calibrate the constants $m$ and $\phi_{g}$. In fact, we find that $n$ is not significantly different from unity, suggesting 
a simple linear relationship, as found previously for cellulose fibers [25], but rather unlike measurements for other flocculated dispersions [6]. However, as for the permeability function, the higher solid fractions of our drainage experiments demand a nonlinear extension of Eq. (8), as described in Appendix A 2 a.

For the solid extensional viscosity $\Lambda$, we adopt the form

$$
\Lambda= \begin{cases}0, & \phi<\phi_{g}, \\ \eta \phi^{2}, & \phi>\phi_{g},\end{cases}
$$

where $\eta$ is a characteristic value. This functional form is crudely based on the phenomenology of collapsing fiber lumen at higher $\phi$ suggested by Ref. [16]. We continue with this form here, in part to assess the significance of such a rate-dependent solid stress, and also to examine how well this functional form reproduces experimental observations at lower $\phi$. Importantly, we assume that Eq. (9) only applies in the gelled state, and include the switch to zero viscosity for $\phi<\phi_{g}$. Nevertheless, at the relatively low solid fractions of our study, the fibers are unlikely to collapse under the imposed loads and the rate-dependent solid stress must have a different microstructural origin, a detail we return to in our conclusions.

The yield condition $|\mathcal{P}|>P_{y}(\phi)$ can be awkward to deal with in situations in which unyielded plugs of solid appear in the suspension. Although one does not expect such plugs to appear in sedimentation tests, they can in fact appear in the drainage problems we consider. In principle, one is then forced to track moving yield surfaces and ensure the plugs remain rigid. Rather than deal with such complications, we adopt a "regularization" of the constitutive model in Eq. (7) similar to to that used for viscoplastic fluid models [26]:

$$
\mathcal{P}=-\left[\frac{P_{y}(\phi)}{\left|\frac{\partial u}{\partial z}\right|+\varepsilon}+\Lambda(\phi)\right] \frac{\partial u}{\partial z},
$$

where $\varepsilon \ll 1$ is a regularization parameter. The law in Eq. (10) applies everywhere; the unyielded regions are taken care of approximately in that, when $|\partial u / \partial z| \ll \varepsilon$, the regularization renders the first term into a relatively large viscouslike stress supplementing $\Lambda(\phi) \partial u / \partial z$. But where $|\partial u / \partial z| \gg \varepsilon$, the regularized law Eq. (10) coincides with the original model Eq. (7). In practice, we take $\varepsilon=10^{-7} \mathrm{~s}^{-1}$, the precise value having been verified as irrelevant.

The regularization of the constitutive law permits a relatively straightforward implementation scheme to solve the model equations numerically. However, the choice $\eta=0$ is inaccessible in this scheme. To access and compare with the rate-independent limit $\eta \rightarrow 0$, and reduce the model to more conventional formulations [6,15], we therefore select a relatively small value for $\eta$ to minimize the effect of the solid viscosity.

\section{B. Ungelled and clear layers}

If $\phi<\phi_{g}, \mathcal{P}=0$ and it then follows from Eqs. (4) and (5) that the pore pressure is hydrostatic and the solid sediments at the local free-fall velocity $V_{\text {sed }}$ :

$$
\frac{\partial p}{\partial z}=-\rho g, \quad \frac{\partial \phi}{\partial t}+\frac{\partial}{\partial z}\left(\phi V_{\text {sed }}\right)=0
$$

and

$$
u=V_{\mathrm{sed}}(\phi, t)=U-\frac{\phi k(\phi) g}{\mu}\left(\rho_{s}-\rho_{f}\right) .
$$

This hyperbolic problem implies that an initially uniform state with $\phi=\phi_{0}<\phi_{g}$ falls uniformly and a shock forms underneath where the solid consolidates to the gel point at height $z=z_{g}(t)$. The ungelled solid also falls away from the top surface leaving behind an overlying clear-fluid layer occupying $z_{f}(t)<z<h$; see Fig. 1(d)(i). In the constant-volume experiments [Fig. 1(a)], the top surface is fixed, so that $h=h_{0}$ and $U$ is set by the imposed background flow. For the drainage 
problems [Figs. 1(b) and 1(c)], that surface falls as water leaks out, and $U=\dot{h}$. The top of the free-fall zone is given by

$$
\dot{z}_{f}=V_{\text {sed }}\left(\phi_{0}, t\right)
$$

or

$$
z_{f}(t)=h(t)+\left[U-\dot{h}-\frac{\phi_{0} k\left(\phi_{0}\right) g}{\mu}\left(\rho_{s}-\rho_{f}\right)\right] t .
$$

Note that there is only a free-fall zone when $z_{f}>z_{g}$; eventually, the freely falling ungelled solid completely falls into the compacting layer, leaving clear fluid above and $z_{f}=z_{g}$ [see Fig. 1(d)(ii)].

\section{Reduction}

Substituting the constitutive law for the solid stress into Eqs. (4) and (5) furnishes an equation for the solid velocity over the consolidated layer where $\phi \geqslant \phi_{g}$ :

$$
u+\frac{k}{\mu} \frac{\partial}{\partial z}\left[\left(\frac{P_{y}}{\left|\frac{\partial u}{\partial z}\right|+\varepsilon}+\Lambda\right) \frac{\partial u}{\partial z}\right]=U-\frac{\left(\rho_{s}-\rho_{f}\right) g}{\mu} k \phi .
$$

This equation must be solved along with the first mass-conservation equation in Eq. (2) subject to the boundary conditions given below.

At the shock at the top of the consolidated layer, $z=z_{g}(t)$, the effective stress vanishes and mass must be conserved, implying

$$
\phi=\phi_{g} \text { and } \frac{\partial u}{\partial z}=0 \text { at } z=z_{g}^{-},
$$

and

$$
\dot{z}_{g}=\frac{\phi\left(z_{g}^{+}, t\right) V_{\mathrm{sed}}\left(\phi\left(z_{g}^{+}, t\right), t\right)-\phi_{g} u\left(z_{g}^{-}, t\right)}{\phi\left(z_{g}^{+}, t\right)-\phi_{g}},
$$

where the \pm superscripts indicate the limits from above and below (respectively). Also, $\phi\left(z_{g}^{+}, t\right)=$ $\phi_{0}$ if $z_{f}>z_{g}$ and there is an overlying free-fall zone, or $\phi\left(z_{g}^{+}, t\right)=0$ if $z_{f}=z_{g}$ and clear fluid overlies the compacting layer.

For fixed-volume compaction as in Fig. 1(a), $h=0$ and $U$ is a prescribed parameter. We then impose $u(0, t)=0$, to complete the formulation of the model for sedimentation with or without flow-through.

For drainage experiments, the net bulk flow $U(t)$ is set self-consistently by the stress conditions at the permeable lower boundary. In particular, there is an outflow condition that dictates the pore pressure at $z=0$ :

$$
p(0, t)=\rho_{f}\left(g h_{\mathrm{exit}}+c U^{2}\right)
$$

where $\rho_{f} g h_{\text {exit }}$ denotes the hydrostatic head at the outflow and $\rho_{f} c U^{2}$ is the resistance to the outflow velocity $U$, modeled using a friction coefficient $c$. Global force balance now demands that the fluid and solid stresses at $z=0$ balance the overlying weight of the suspension:

$$
\mathcal{P}(0, t)+p(0, t)=\rho_{f} g h+\phi_{0} h_{0}\left(\rho_{s}-\rho_{f}\right) g .
$$

As a consequence,

$$
\mathcal{P}(0, t)+\rho_{f} c U^{2}=\rho_{f} g\left(h-h_{\text {exit }}\right)+\phi_{0} h_{0}\left(\rho_{s}-\rho_{f}\right) g,
$$

which completes the model for drainage. 


\section{Capillary stresses for drainage and freeness}

If the water drains from the chamber, then the model outlined above applies only as long as $z_{g}<h$ and there is an ungelled or clear-fluid layer above the compacting region. However, the fluid surface eventually descends to $z=z_{g}(t)$. Any subsequent drainage must either expose a dry solid phase or prompt capillary stresses to appear that maintain contact between the solid and fluid surfaces. In the experiments, the top surface always remains wet, with no evidence of an overlying unsaturated solid. Therefore, we assume that capillary stresses act to hold the fluid and solid surfaces together once they meet, and $z_{g}=h$ [see Fig. 1(d)(iii)]. This demands that we abandon the upper boundary conditions, $\phi=\phi_{g}$ and $\partial u / \partial z=0$ at $z=z_{g}^{-}$, and instead apply

$$
u\left(z_{g}^{-}, t\right)=\dot{z}_{g}=\dot{h} .
$$

A similar situation arises when the initial suspension is already gelled, $\phi_{0}>\phi_{g}$, and the water and solid surfaces coincide at $t=0$. Such initial states turn out to characterize the freeness test for the pulp suspension we employ in our experiments, which begin from a well-mixed initial condition that is slightly above the gel point. In this situation, and without any interfacial interaction, the phases separate at the top under sedimentation. But by including capillary effects, we may again prevent the water surface from detaching from the top of the solid.

\section{EXPERIMENTAL DETAILS}

To calibrate the compressive yield stress $P_{y}(\phi)$ and permeability $k(\phi)$ and then gauge the importance of any rate-dependent solid stress, we perform laboratory experiments using northern bleached softwood Kraft (NBSK) pulp suspended in water (obtained from Canfor Pulp). NBSK pulp is produced from a blend of tree species (pine, spruce, and fur); the fibers have a mean length and equivalent diameter of $2.6 \mathrm{~mm}$ and $26 \mu \mathrm{m}$, respectively, as measured by a fiber quality analyzer (Optest) [16,27].

In a first series of tests, we conducted sedimentation experiments in a selection of three cylinders with interior diameters of $10.0,13.6$, and $19.0 \mathrm{~cm}$. The initial depth of the suspension $h_{0}$ was varied from 3.1 to $88.3 \mathrm{~cm}$. From these tests, we measured the final sedimented height; i.e., the level of the top surface of solid. As described in Sec. IV, this measure can be used to calibrate $P_{y}(\phi)$.

The second set of tests used a tank composed of a large rectangular chamber and a water reservoir as sketched in Fig. 1(a). The suspension was placed in the chamber and was confined between screen meshes. Water could be recirculated between the reservoir and test chamber by a pump, providing a closed flow-loop. In this second arrangement, we first performed more sedimentation tests without flow-through $(U=0)$ to supplement the final height data from the cylinders and further constrain $P_{y}(\phi)$. We then activated the pumps and measured the effect of the additional flow-induced compaction on the final solid height, which allows us to calibrate the permeability (Sec. V). The rectangular chambers had a cross-section of $15.2 \mathrm{~cm}$ by $14.0 \mathrm{~cm}$, and the depth of the pulp compartment was $h_{0}=28.2 \mathrm{~cm}$. Flow speeds in the range $2.26 \times 10^{-5} \mathrm{~m} / \mathrm{s}$ to $2.07 \times 10^{-4} \mathrm{~m} / \mathrm{s}$ were attained. A variant of the flow-through experiments, in which water was drained from the arrangement at fixed rate, also allowed a cruder estimation of the compressive yield stress at higher solid fraction (Appendix A 1).

For the flow-through tank, we also recorded high resolution camera images of the compacting suspension through the sidewall during the experiments. From these images we measured the instantaneous height of the top surface of the solid, $z_{f}(t)$. We also seeded the pulp with small pieces of black paper (of a few $\mathrm{mm}$ in size) to act as tracers for one-dimensional particle image velocimetry (PIV). The PIV extracts the horizontally averaged vertical displacements between consecutive frames, which were spaced by $10 \mathrm{~s}$ in these tests with relatively slow flow speeds. Both measures of the time-dependent dynamics allowed us to quantify the transient adjustments that took place after the suspension was mixed up and left to settle (with or without flow-through), and when the pump was suddenly switched on or changed in flow rate. 

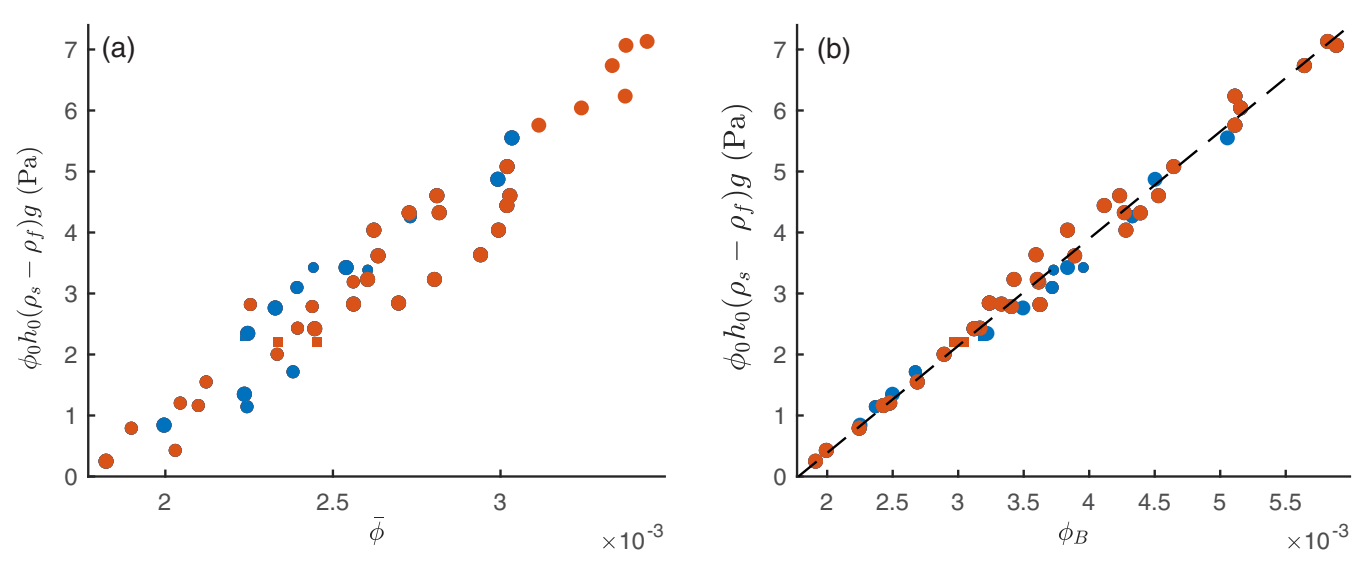

FIG. 2. Results of the sedimentation experiments, plotting (in (a)) the total gravitational stress on the solid, $\left(\rho_{s}-\rho_{f}\right) g \phi_{0} h_{0}$, against the average final solid fraction, $\bar{\phi}=\phi_{0} h_{0} / h_{f}$. This data is replotted in (b) as $P_{y}\left(\phi_{B}\right)$ against $\phi_{B}$, using Eqs. (24) and (25), having fitted the parameters $m=1.756 \times 10^{3} \mathrm{~Pa}$ and $\phi_{g}=0.00178$ according to the procedure outlined in Sec. IV; the fit itself is shown by the dashed line. The different symbols refer to the two batches of pulp with different $\phi_{0}$ (blue for $\phi_{0}=0.00165$ and red for $\phi_{0}=0.00167$ ), and the different containers (circles for the cylinders, with size corresponding to radii; squares for the rectangular flow-through tank).

A third suite of experiments focused on the time-dependent dynamics of drainage tests in a large, open top rectangular tank within which the pulp was suspended above a permeable screen; see Fig. 1(b). The bottom of the tank was connected to an outlet pipe ending in a tap fixed at an adjustable vertical position that was below the initial top surface of the suspension. The height of the tap sets a net hydrostatic pressure drop across the system that forces water within the pulp chamber to drain to a given level, thereby compacting the solid against the screen. The initial height of the suspension in the drainage tank was $h_{0}=41.9 \mathrm{~cm}$; the tank's cross-sectional area was approximately $390 \mathrm{~cm}^{2}$. The exit pipe was $1 \mathrm{~m}$ long and $2.54 \mathrm{~cm}$ in diameter and its end was held at a range of heights from $28.5 \mathrm{~cm}$ down to $3.5 \mathrm{~cm}$. The resistance coefficient was measured to be $c=3.44 \times 10^{4}$ from steady flow-through tests with pure water. Again, we use the black paper tracers to perform PIV for the arrangement; this time the images were spaced by 1 second in view of the faster flow speeds that were achieved. Both the drainage tests and the transient adjustments in the flow-through tank are used to gauge the importance of any solid viscosity $\Lambda(\phi)$ in Sec. VI.

\section{SEDIMENTATION TESTS: CALIBRATION OF $P_{\mathbf{y}}(\phi)$}

For a vertical tube filled with a suspension of solid fraction $\phi_{0}$ to a height $h_{0}$, sedimentation proceeds until the total weight of solid material $\left(\rho_{s}-\rho_{g}\right) g \phi_{0} h_{0}$ is balanced by the compressive yield stress at the bottom, $P_{y}\left(\phi_{B}\right)$. Moreover, in the steady state, the gravitational stratification of the solid dictates the final height $h_{f}$, establishing a connection with the base solid fraction $\phi_{B}$ that provides us the means to calibrate the compressive yield stress. For the task, we conducted sedimentation tests in the three cylinders and the rectangular tank of the flow-loop arrangement, beginning with initial concentrations below $\phi_{g}$. For equivalent selections of $\phi_{0}$ and $h_{0}$, the final settled height was consistent across all four containers. However, the time taken to reach the final settled height was significantly larger in the smallest diameter cylinder than for the other containers, for which the sedimentation rates were similar.

Figure 2(a) shows the average final solid fraction, $\bar{\phi}=\phi_{0} h_{0} / h_{f}$, against the total gravitational stress on the solid, $\left(\rho_{s}-\rho_{f}\right) g \phi_{0} h_{0}$. When the final sedimented state is almost uniform, that overburden is balanced by $P_{y}(\bar{\phi})$. Thus, aside from any gravitational stratification, the plot in 
Fig. 2(a) reflects the compressive yield stress function. Although there is some spread, the data are suggestive of a linear dependence of $P_{y}(\phi)$ on solid fraction, which guides us to take $n=1$ in (8) for a deeper analysis of the experimental results. Further evidence for this choice is provided later.

For $P_{y}(\phi)=m\left(\phi-\phi_{g}\right)$ and no flow-through $(U=0)$, the steady-state equations reduce to

$$
\frac{d \mathcal{P}}{d z}=m \frac{d \phi}{d z}=-\left(\rho_{s}-\rho_{f}\right) g \phi
$$

which, given that $\phi=\phi_{g}$ at $z=h_{f}$, has solution

$$
\phi=\phi_{g} \exp \left[\frac{g}{m}\left(\rho_{s}-\rho_{f}\right)\left(h_{f}-z\right)\right] .
$$

The base solid fraction is therefore

$$
\phi_{B}=\phi_{g} e^{\left(\rho_{s}-\rho_{f}\right) g h_{f} / m} .
$$

The integral of Eq. (22) across the height of the solid also provides the global force balance condition,

$$
P_{y}\left(\phi_{B}\right)=m\left(\phi_{B}-\phi_{g}\right)=\left(\rho_{s}-\rho_{f}\right) g \phi_{0} h_{0},
$$

which leads to a prediction for the final settled height,

$$
h_{f}=\frac{m}{g\left(\rho_{s}-\rho_{f}\right)} \log \left[1+\frac{\left(\rho_{s}-\rho_{f}\right) g \phi_{0} h_{0}}{m \phi_{g}}\right] .
$$

The constant $\left(\rho_{s}-\rho_{f}\right) g \approx 5 \times 10^{3} \mathrm{~kg} / \mathrm{m}^{2} / \mathrm{s}^{2}$, and each experiment furnishes a pair $\left(\phi_{0} h_{0}, h_{f}\right)$. We therefore determine the values for $m$ and $\phi_{g}$ that best approximates this equation in a least squares sense over all 49 experiments. We find $m=1.756 \times 10^{3} \mathrm{~Pa}$ and $\phi_{g}=0.00178$.

The fitted linear compressive yield stress function is plotted in Fig. 2(b) along with the experimental data for $P_{y}\left(\phi_{B}\right)$ and $\phi_{B}$, implied by Eqs. (25) and (24), given the fitted values of $m$ and $\phi_{g}$. The agreement between the model fit and the experimental data is satisfactory, leading us to conclude that $P_{y}(\phi)$ is well represented by a linear function near the gel point. This conclusion is reinforced by repeating the analysis, but including the exponent $n$ as a further free parameter: the nonlinear least squares fit to the generalization of Eq. (26) predicts that $n \approx 0.93$. This fitted exponent is not significantly different from unity given that it leads to no qualitative change to Fig. 2. In particular, the root-mean-square (RMS) error in Eq. (26) normalized by the observed $h_{f}$ is reduced from $4.55 \%$ to $4.52 \%$ upon changing $n$ from 1 to 0.93 .

\section{FLOW-ASSISTED COMPACTION TESTS: CALIBRATION OF $k(\phi)$}

The permeability function can be calibrated by measuring the steady-state solid heights for given bulk flow velocities $U$ in the flow-through tank. To build such a data set, we first mixed the suspension and then let the solid settle to steady state without any flow-through $(U=0)$. The pumps were then turned on and the rate increased sequentially, waiting at each pump setting for the steady state to be reached. The settled heights obtained in this way are plotted against $U$ in Fig. 3(a).

In steady state flow-through the force balance equation becomes

$$
m \frac{d \phi}{d z}=\frac{\mu U}{k(\phi)}-\left(\rho_{s}-\rho_{f}\right) g \phi
$$

which, for a nearly uniform suspension, indicates that

$$
k(\bar{\phi}) \sim \frac{\mu U h_{f}^{2}}{\phi_{0}^{2} h_{0}^{2}}\left[2 m\left(1-\frac{\phi_{g} h_{f}}{\phi_{0} h_{0}}\right)-\left(\rho_{s}-\rho_{f}\right) g\right]^{-1} .
$$



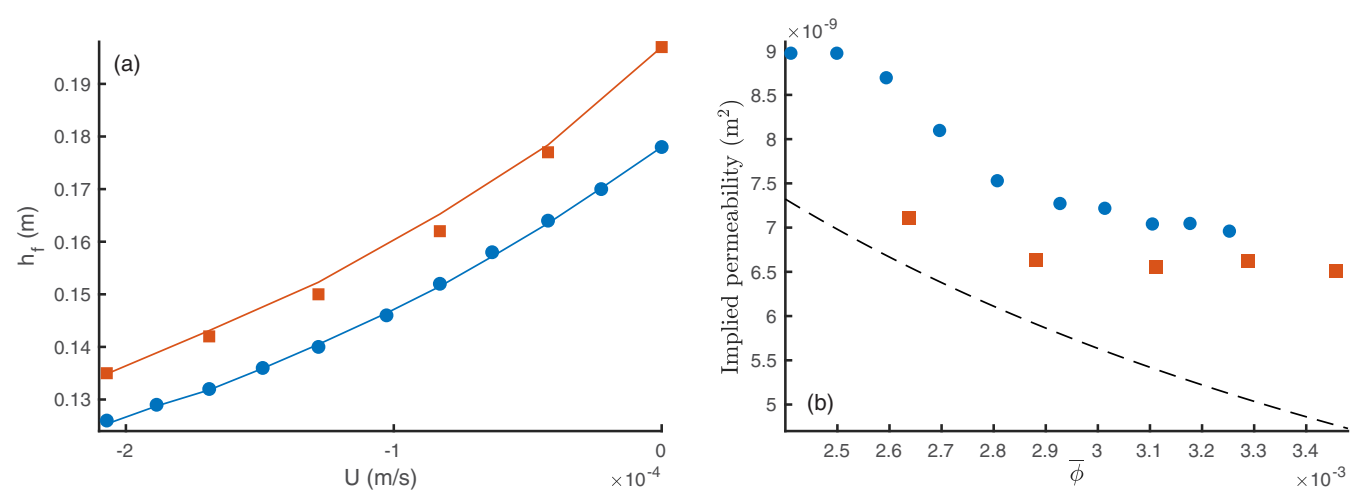

FIG. 3. (a) Settled heights $h_{f}$ against flow velocity $U$, showing both the experimental results (filled squares and circles) and the theoretical predictions (solid lines), given the fits established in Sec. V. (b) The implied mean permeability for a nearly uniform suspension given by Eq. (28), plotted against $\bar{\phi}=\phi_{0} h_{0} / h_{f}$; the dashed line shows Eq. (6) with the calibrated $A$ and $\alpha$. The (blue) circles have $\phi_{0}=0.0015$ and are used in the fits for $A$ and $\alpha$; the (red) squares show an independent data set with $\phi_{0}=0.0017$.

Figure 3(b) plots the implied $k(\bar{\phi})$ against $\bar{\phi} \equiv \phi_{0} h_{0} / h_{f}$ for our steady-state flow-through experiments. This data suggests that the main dependence of the permeability on the solid fraction is through a factor $\phi^{-1}$, as in the fit (6) and found by previous studies [25].

With the adopted form $k(\phi)=A \phi^{-1} \log (\alpha / \phi)$, the implicit solution to Eq. (27) is

$$
h_{f}-z=-\frac{m A \Gamma}{\mu U} \log \left[\frac{\phi}{\phi_{g}}\left(\frac{\Gamma-\log (\phi / \alpha)}{\Gamma-\log \left(\phi_{g} / \alpha\right)}\right)^{\Gamma}\right],
$$

where $\Gamma=-\mu U /\left[\left(\rho_{s}-\rho_{f}\right) g A\right]$. The global stress balance obtained by integrating Eq. (27) now gives

$$
e^{-\Gamma}\left(\frac{\phi_{g}-\phi_{B}}{\Gamma \alpha}-\frac{\mu U}{m A \Gamma^{2} \alpha} \phi_{0} h_{0}\right)=E_{1}\left[\Gamma-\log \left(\frac{\phi_{g}}{\alpha}\right)\right]-E_{1}\left[\Gamma-\log \left(\frac{\phi_{B}}{\alpha}\right)\right],
$$

where $E_{1}(x)=\int_{x}^{\infty} e^{-t} / t d t$ is an exponential integral and $\phi_{B}$ follows from Eq. (29) with $z=0$. We use this expression to fit $A$ and $\alpha$ in a least squares sense, given the previously determined values of $m$ and $\phi_{g}$. This procedure leads to $A=3 \times 10^{-12} \mathrm{~m}^{2}$ and $\alpha=0.84$. The effectiveness of the fit can be judged by Fig. 3, which includes the theoretical predictions for the final height (given the fitted values of the parameters). The figure contains two sets of data with slightly different $\phi_{0}$; only one of these sets (shown with blue circles) was used to fit $A$ and $\alpha$. Given those parameter settings, the second set of data is reproduced with an RMS error (normalized by the observed $h_{f}$ ) of $1.23 \%$; the first set has error $0.32 \%$.

The fidelity of the fit can be further justified by using a more general permeability function of the form $A \phi^{-\ell} \log (\alpha / \phi)$ and varying $\ell$. We find that $\ell=1$ provides a superior fit than any other power larger than 1.1 or less than 0.9 ; the RMS error when $\ell=1.1$ or 0.9 is larger than that for $\ell=1$ by around $30 \%$.

Note that the pump rate was only increased in steps in this series of experiments. With a reduction of the flow rate, the suspension shows highly hysteretic behavior, with very little recovery and expansion to a less consolidated structure. In the most extreme case of turning off a test with the highest pump rate, the top surface of the solid rebounds upwards by at most a millimeter or two. By comparison, when that pump rate is switched on to compress the gravitationally sedimented state, the top surface is pushed down by around $7 \mathrm{~cm}$. Thus, at least for the degree of consolidation 

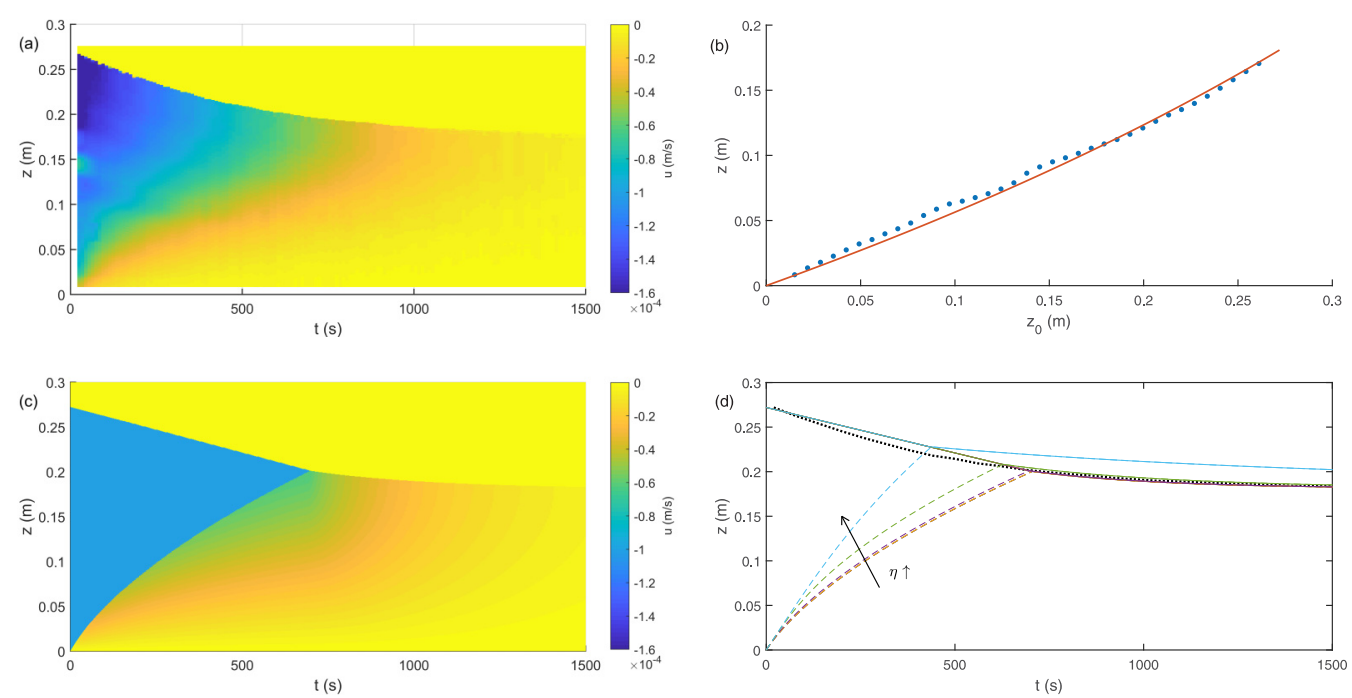

FIG. 4. Dynamics of a sedimentation experiment with $\phi_{0}=0.00154$ and $h_{0}=0.272 \mathrm{~m}$. Shown are plots of the solid velocity as a density on the $(t, z)$ - plane for (a) experimental PIV and (c) a model solution with $\eta=\eta_{*}=10^{7} \mathrm{~Pa}$ sec. In (b), the PIV displacements are integrated to determine the final positions of tracers that were uniformly distributed through the column (dots), with solid line showing the corresponding theoretical prediction. Panel (d) shows time series of the interface $z_{f}$ (solid) and gel height $z_{g}$ (dashed) for further theoretical solutions with $\eta / \eta_{*}=10^{-3}, 10^{-2}, 0.1,1,10$ and 100 ; the dotted line shows the experimental observation of $z_{f}$.

experienced in the current tests, the fiber matrix must deform almost plastically on compression, as assumed in the model.

\section{DYNAMICS AND THE RATE-DEPENDENCE OF THE SOLID STRESS}

\section{A. Fixed-volume compaction dynamics}

We begin our assessment of the time-dependent dynamics predicted by the model with the transient dynamics observed in the flow-through tank. Figures 4(a) and 5(a) show the solid velocity measured by PIV during fixed-volume sedimentation experiments either without flow-through $\left[U=0\right.$; Fig. 4(a)] or assisted with $U=-2.07 \times 10^{-4} \mathrm{~m} / \mathrm{s}$ [Fig. 5(a)]. The figures compare these results with model predictions, computed using the calibrations of $P_{y}(\phi)$ and $k(\phi)$, described in Secs. IV and V, bridged to higher solid fractions as summarized in Appendix A. For the rate-dependent solid stress, we use the bulk viscosity in Eqs. (7) and (9), leaving $\eta$ as a variable parameter. Note that pressure filtration studies [16] suggest that $\eta \approx 10^{7} \mathrm{~Pa}$ sec for NBSK, a parameter setting that we denote by $\eta_{*}$ (in Ref. [16], this material parameter was written as $\lambda_{*}$, the inverse of $\eta_{*}$ ).

The model solutions are weakly sensitive to the value of $\eta$ in these calculations, as illustrated by the time series of $z_{f}(t)$ and $z_{g}(t)$ for different choices of the bulk viscosity in Figs. 4(d) and $5(\mathrm{~d})$; only for relatively large values of $\eta$ (in comparison to $\eta_{*}$ ) is there any suggestion that the rate-dependent solid stress participates in the settling dynamics. Moreover, the comparison with the experimental PIV, which is otherwise qualitatively successful, implies that such cases are not realistic. The insignificance of the bulk solid viscosity in these tests for $\eta=O\left(\eta_{*}\right)$ can be established more directly by dimensional analysis with the model equations: in comparison to the relative velocity $u-\dot{h}$, the viscous term on the left of Eq. (15) is of order $\eta_{*} \phi_{g}^{2} k\left(\phi_{g}\right) /\left(\mu h_{0}^{2}\right)=O\left(10^{-3}\right)$, if we use the initial height $h_{0}$ and gel fraction $\phi_{g}$ as characteristic scales for length and solid fraction. 

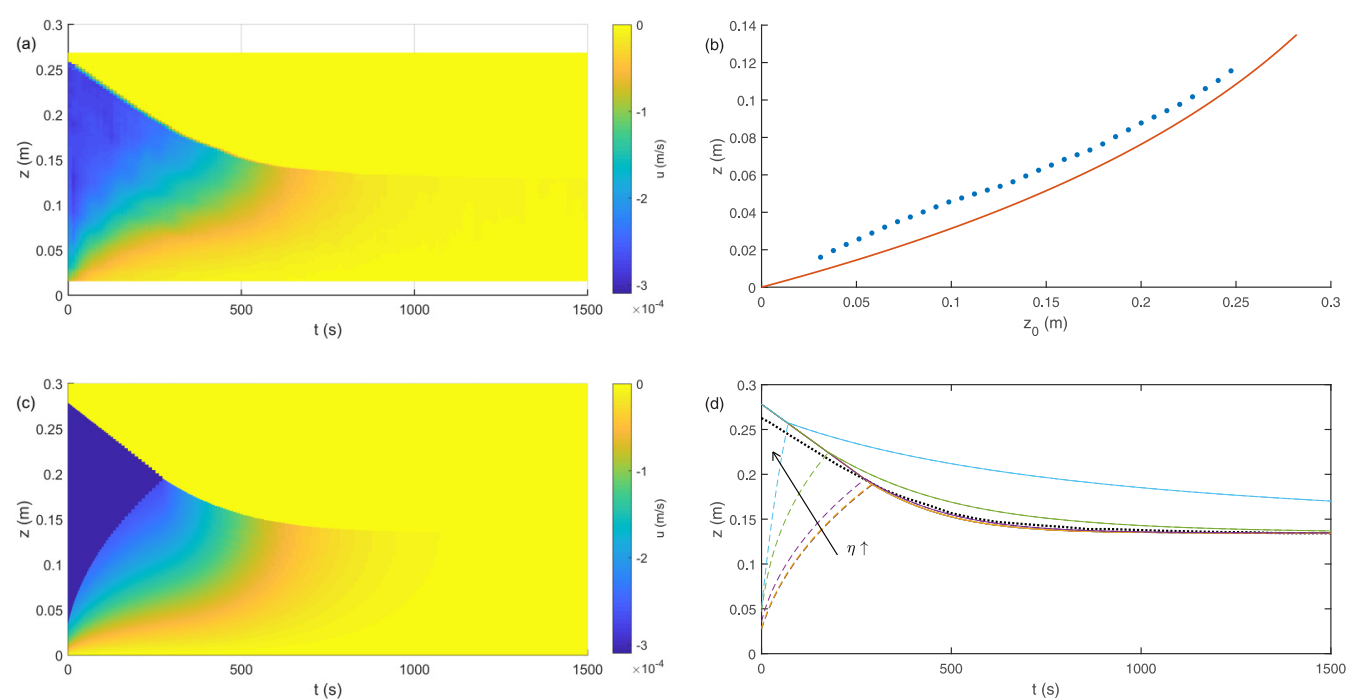

FIG. 5. A similar set of plots as in Fig. 4, but for a flow-assisted sedimentation experiment with $U=-2.07 \times 10^{-4} \mathrm{~m} / \mathrm{s}, \phi_{0}=0.00166$ and $h_{0}=0.282 \mathrm{~m}$.

Our fixed-volume compaction tests can therefore be adequately reproduced by the two-phase model with just a compressive yield stress, as found by previous studies (e.g., Ref. [8]).

A different comparison of theory and experiment is given by the $\phi$-distribution of the final steady state. This distribution is, however, difficult to extract from the PIV measurements because of the need to differentiate the particle displacements in $z$ : in our one-dimensional approximation of the compaction problem, conservation of mass demands that the solid fraction at any time $t$ be related to the initial distribution by

$$
\frac{d z}{d z_{0}}=\frac{\phi\left(z_{0}, 0\right)}{\phi(z, t)}=\frac{\phi_{0}}{\phi(z, t)},
$$

where $z_{0}$ is the the initial position of an element of solid. The mapping from initial to final positions of tracers encoded in $z\left(z_{0} ; t\right)$ provides an alternative means of comparing the theory and experiments. For example, for sedimentation without flow-through $(U=0)$,

$$
z=\frac{m}{\left(\rho_{s}-\rho_{f}\right) g} \log \left[\frac{m \phi_{g}+\left(\rho_{s}-\rho_{f}\right) g \phi_{0} h_{0}}{m \phi_{g}+\left(\rho_{s}-\rho_{f}\right) g \phi_{0}\left(h_{0}-z_{0}\right)}\right],
$$

corresponding to Eq. (23). The mapping $z=z\left(z_{0} ; t\right)$ is conveniently extracted from the experiments using the cumulative displacements between consecutive images found by PIV. Figures 4(b) and 5 (b) include the experimentally measured and theoretically predicted final profiles of $z\left(z_{0}\right)$ for those two particular experiments. Overall, the experiment and theory are in agreement (with the example in Fig. 5 being one of the more demanding cases), providing further confidence in our fits of the constitutive functions $P_{y}(\phi)$ and $k(\phi)$.

Despite the qualitative agreement of theory and experiment in Fig. 4, there is a notable difference between the two over in the free-fall zone $z_{g}(t)<z<z_{f}(t)$ which, according to the model, should contain solid with the initial fraction $\phi_{0}$ falling at the constant free-fall velocity $V_{\text {sed }}\left(\phi_{0}, t\right)$. By contrast, the experimental PIV data indicate a variable fall velocity over this region, which was also noticeable in the descent of the solid surface $h(t)$ [see Fig. 4(d)], and evident in all our experiments. A variable fall speed may arise because a well-mixed initial condition is hard to establish in the experiments, furnishing a non-uniform initial solid fraction. Indeed, assuming that the final sedimented state is given by Eq. (23), one can use Eq. (31) and the PIV data to trace $\phi$ back to 


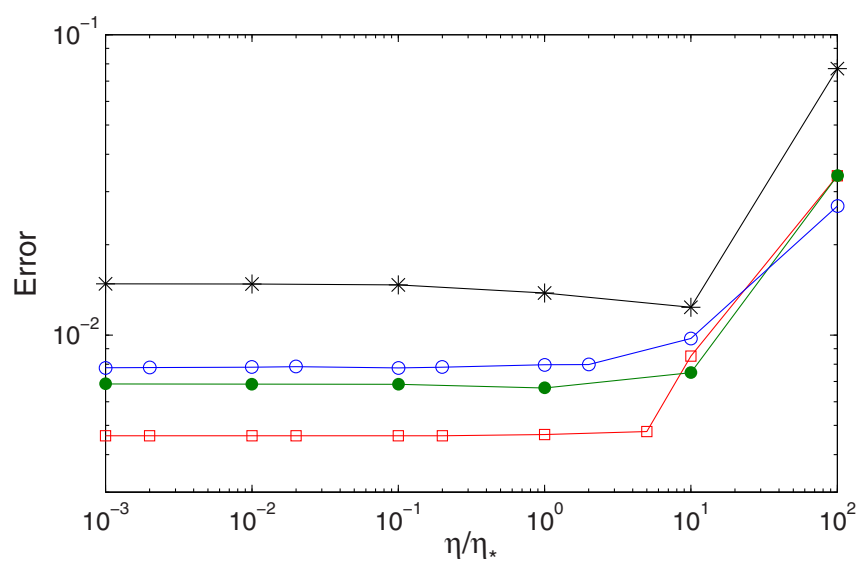

FIG. 6. Root-mean-square error in the interface position $z_{f}(t)$, normalized by its mean value for a number of sedimentation and flow-through experiments. These experiments include an example of pure sedimentation (red squares), a test in which sedimentation was assisted with the largest bulk flow rate $\left(U=-2.07 \times 10^{-4} \mathrm{~m} / \mathrm{s}\right.$; black stars), and tests in which the solid was allowed to sediment before turning the pumps on. For the latter, two cases are shown: one in which the pumps were again turned to the maximum (green filled circles), and a second in which the pump rate was increased in five steps up to that maximum (blue open circles; this test corresponds the squares in Fig. 3).

where the solid was ungelled and in free fall. This procedure implies an initial state characterized by irregular spatial structure with $\phi_{0}=0.0017 \pm 0.0008$.

It is also possible that a non-monotonic sedimentation flux could generate a non-uniform free-fall zone, as suggested previously for other suspensions [28,29]. However, one still expects a uniform region embedded within that zone (if $\phi(z, 0)=\phi_{0}$ ), unlike what is seen in Fig. 4(a). Moreover, although the spatial differentiation of the PIV measurements introduces significant noise in the local estimates of $\phi$, the same data also suggests that the sedimentation flux is not a simple function of the local solid fraction. The discrepancy may well therefore point to some other behavior in the free-fall zone that is not accounted for in the model (such as spatial inhomogeneity induced by the flocculation of fibers, or the additional drag experienced by the markers nearer the side walls). Awkwardly, this feature also precludes us from exploiting the PIV data to directly infer reliable permeability data from the sedimentation experiments.

A measure of the goodness-of-fit of the theoretical model over all of the sedimentation and flowthrough experiments is shown in Fig. 6. This figure plots the root-mean-square error in the interface position $z_{f}(t)$ for the model using a spread of values of $\eta$. The error is normalized by the mean interface position and, unless the solid viscosity is chosen to be excessively high, is of the order of a few percent.

\section{B. Drainage}

The higher fall speeds achieved during the drainage experiments provide a more demanding test of the model and an indication as to whether or not a solid viscous stress is needed: in the most extreme case with $h_{\text {exit }}=0.035 \mathrm{~m}$, the mean solid fraction reaches approximately 0.02 and the solid speed peaks at around $1 \mathrm{~cm} / \mathrm{s}$ (about four times denser and 50 times faster than in the constant-volume tests). Importantly, in the drainage tests, the relatively high resistance of the output pipe has the effect of throttling the fall speed of the water. Consequently, the water height $h(t)$ is largely set by the "pipe law" $c \dot{h}^{2} \approx g\left(h-h_{\text {exit }}\right)$, in all but the most extreme cases of $h_{\text {exit }}$ where the densification of the solid can offset the pipe resistance. Moreover, in most cases, the interface $z_{f}(t)$ does not have sufficient time to sediment below the free surface to create an observable clear layer 

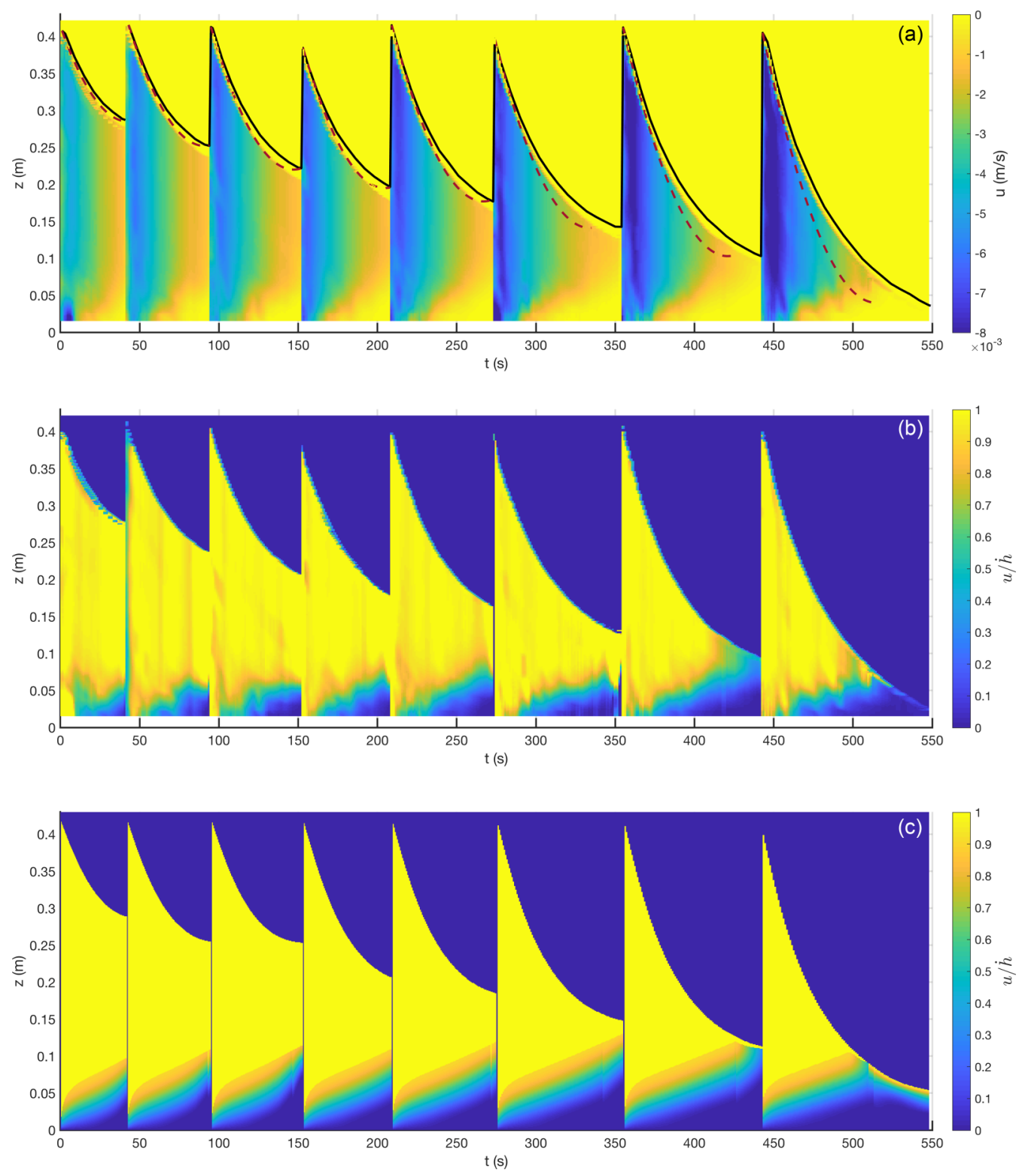

FIG. 7. (a) Solid velocity (in $\mathrm{m} / \mathrm{s}$ ) obtained from PIV of eight drainage tests, plotted in series. The tests drain to the heights $h_{\text {exit }}$ terminating each case. Also shown is the experimentally observed water height $h(t)$ (solid black) and the solution to the pipe law $c \dot{h}^{2}=\rho_{f} g\left(h-h_{\text {exit }}\right)$ (dashed red). In panel (b) we replot the data using the scaling $u(x, t) / \dot{h}(t)$. In panel (c), we show the theoretical counterpart to panel (b) using the solid extensional viscosity $\eta=\frac{1}{2} \eta_{*}$.

at the top. Thus, the time series of both $h(t)$ and $z_{f}(t)$ are not the best statistics to gauge the solid extensional viscosity. Instead, we use the growth of the gelled layer at the base of the column as a clearer diagnostic.

Figure 7(a) shows the solid velocities obtained by PIV for a number of drainage experiments with varying $h_{\text {exit }}$ starting from $h_{0}=0.419 \mathrm{~m}$ with $\phi_{0}=0.0016$. Except for the cases with small $h_{\text {exit }}$, the water heights follow the parabolas predicted by the pipe law, and the bulk of the solid falls 
at roughly uniform velocity given by $\dot{h}$. More significant is the sharp decline of the fall speed into the gelled layer underneath, which is highlighted by plotting the fall velocity normalized by the free surface velocity, $u(x, t) / \dot{h}[$ Fig. 7(b)]; although the division introduces some noise, the free-fall zone and compacted layer become more apparent. Figure 7(c) adds complementary model computations of $u(x, t) / \dot{h}$ using a solid extensional viscosity parameter of $\eta=\frac{1}{2} \eta_{*}$, a choice that is motivated below.

Figure 8 shows further details of the theoretical solutions for one of the tests. Computations with different bulk viscosities $\eta$ are shown, illustrating how the position of the top surface is not particularly sensitive to this parameter. The degree of compaction at the base of the column is, however, controlled by $\eta$, with low bulk viscosities generating a compacted layer bordered from the overlying falling zone by a relatively sharp interface with pronounced velocity gradients. Raising the solid extensional viscosity smooths these gradients to furnish a more gradual transition. Furthermore, bulk viscosities close to the value suggested by pressure filtration, $\eta=\eta_{*}$, lead to velocity gradients that are more consistent with the PIV observations. Thus, the qualitative comparison of the PIV and theoretical velocity plots, and in particular the transition above the compacted layer, indicates that the model performs better with a solid extensional viscosity $\eta=O\left(\eta_{*}\right)$ than without one.

A more quantitative evaluation of this conclusion is given in Fig. 9, which reports, for all the drainage tests, the root-mean-square error in the free surface height $h(t)$ (again normalized by the mean surface height) and the average distance between the contours for which $u(z, t) / \dot{h}=0.5$ and 0.8 ; see Fig. 8. The latter is a direct measure of the sharpness of the transition in solid velocity above the compacted layer and has the curious feature of being roughly independent of time and $h_{\text {exit }}$ for both the experiments and model solutions. The plots of this diagnostic in Fig. 9(b) suggest that that the model fits the experiments best for bulk viscosities close to $\eta_{*}$; a near-optimal choice indicated by Fig. 9(b) is $\eta=\frac{1}{2} \eta_{*}$, as used in Fig. 7(c).

Note that the PIV detects some degree of rebound of the solid once the water drainage terminates: although there is no change in the position of the top surface of the solid, fibers near the base of the column deform back upwards over distances of up to a few millimeters over times of order tens of seconds. We interpret this rebound to be the signature of recovery from a small amount of elastic stress superposed on the plastic compression. As the model incorporates no such dynamics, we have avoided any comparisons with the PIV after the termination of the drainage.

\section{UNDERSTANDING FREENESS}

Equipped with a calibrated two-phase model for a specific suspension of cellulose fibers, we now turn to an exploration of the Canadian Standard Freeness test. In particular, in addition to verifying that the calibrated model reproduces the freeness score for the pulp suspension, we explore the dynamics of the test to gauge what material metric the freeness score provides for a two-phase medium.

\section{A. Freeness scores}

The arrangement of the Canadian Standard Freeness (CSF) test is sketched in Fig. 1(c): a suspension of pulp held in a cup drains through a thin permeable screen into a funnel; a side channel diverts part of the discharge into a collection tube to register the "freeness score" (in ml). Because the build-up of solid above the screen limits the drainage, the freeness score represents a nonlinear integral measure of the drainage dynamics. Under standard conditions, the tester is initialised with $1 \mathrm{~L}$ of the suspension (leading to $h_{0} \approx 0.122 \mathrm{~m}$ ) with a consistency of $0.3 \%$-byweight (corresponding to $\phi_{0} \approx 0.002$, if $\rho_{s}=1500 \mathrm{~kg} / \mathrm{m}^{3}$ and $\rho_{f}=1000 \mathrm{~kg} / \mathrm{m}^{3}$ ). Clear water has a freeness score in the range 880 to $890 \mathrm{ml}$.

To compute theoretical predictions for the freeness score, we supplement the theory in Sec. II with a model of the funnel that determines how much of the discharge from the cup enters the side 

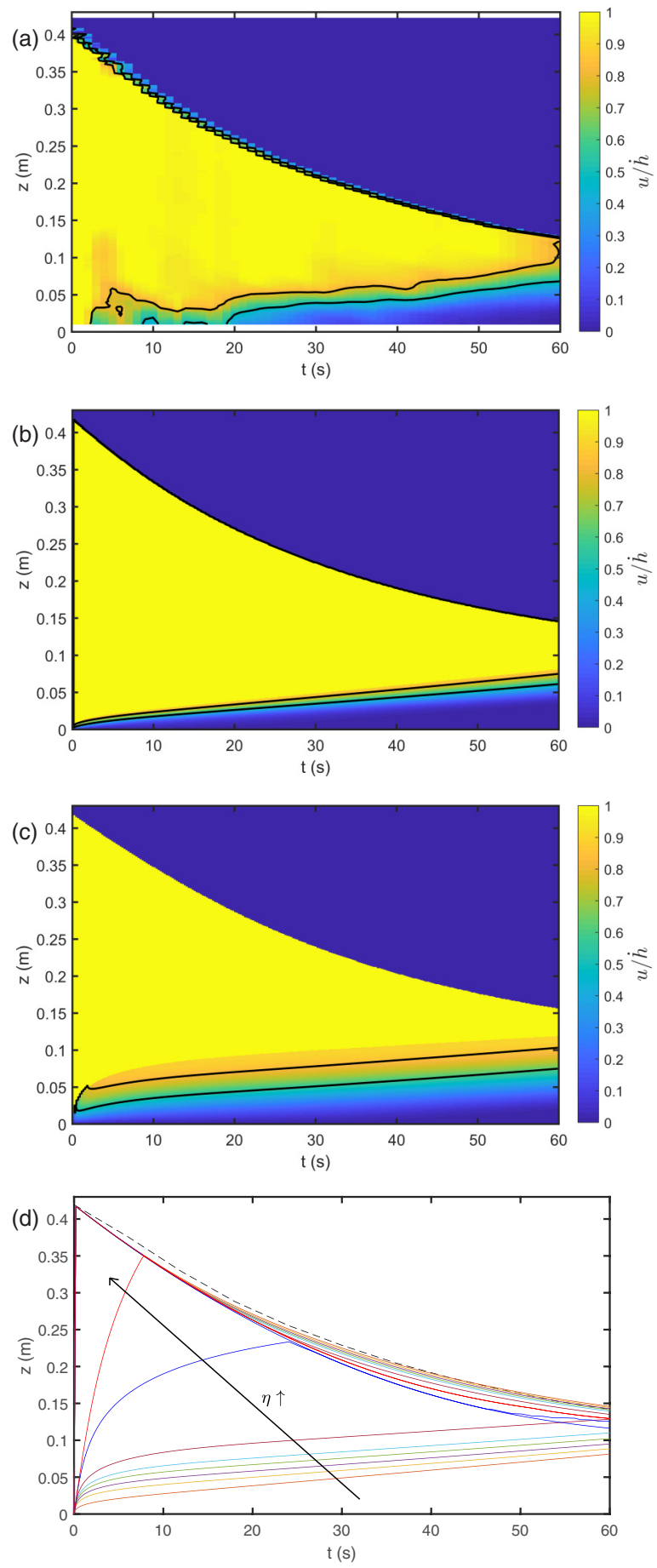

FIG. 8. A drainage test with $h_{\text {exit }}=10.3 \mathrm{~cm}$. Panels (a)-(c) show plots of the scaled solid velocity $u / \dot{h}$ as densities over the $(z, t)$ - plane for (a) the experimental PIV, and model solutions with (b) $\eta=10^{-3} \eta_{*}$ and (c) $\eta=\eta_{*}$. The lines indicate the contours along which $u / \dot{h}=0.5$ and 0.8 . Panel (d) plots time series of the interfaces $h \approx z_{f}$ and $z_{g}$ for solutions with $\eta / \eta_{*}=10^{-3}, 10^{-2}, 0.1,0.2,0.333,0.5,1,10$, and 100; the dashed line shows the free surface of the experiment. 

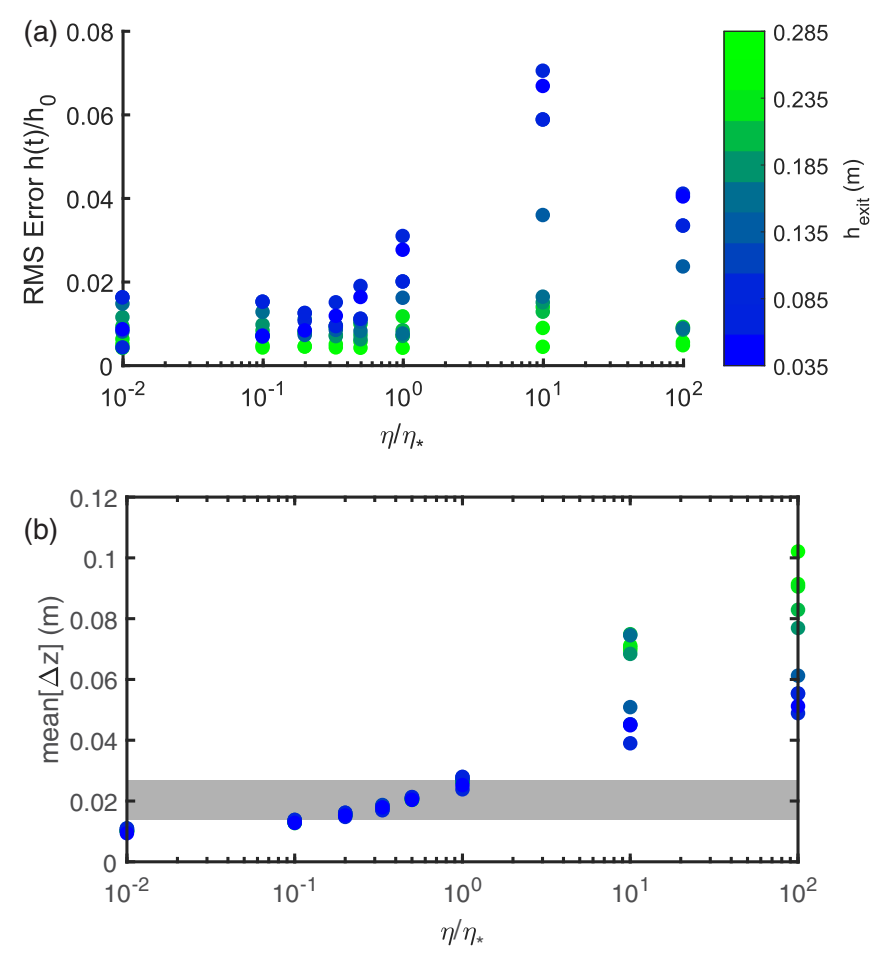

FIG. 9. (a) Root-mean-square error in $h(t)$ and (b) the average distance between the contours along which $u(z, t) / \dot{h}=0.5$ and 0.8 ; see Fig. 8. In panel (a), the error is normalized by $h_{0}$. In panel (b), the dots show model predictions; the grey shaded region indicates the range of experimental measurement as defined from the cumulative distribution of observed values (the distribution is strongly skewed; we use the limits within which $68 \%$ of the data lie). The color bar in panel (a) maps the color of the dots in both panels to $h_{\text {exit }}$.

channel. We relegate the details of that extension to Appendix B, its sole purpose being to convert the flow history to the freeness score.

Because the permeable screen of the cup presents less resistance to outflow than the pipe of our drainage tests (the resistance coefficient of the screen is estimated to be $c=180$, in comparison to the pipe coefficient $c=3.4 \times 10^{4}$ ), the dynamics of the freeness score are richer than the experiments in Sec. VI. Thus, as well as performing a further verification of the model and its calibration, the freeness test serves up a potentially informative application of the model. However, a single score for a given pulp suspension is limiting, leading us to perform a series of freeness tests in which we departed from standard procedure and varied the amount of pulp in the cup and its initial solid fraction.

The results of these tests are displayed in Fig. 10; the freeness score shows a remarkably linear dependence on the initial weight of pulp, over a range of initial consistencies. Both the values of freeness and the trend with initial weight and consistency are recovered by the model when the solid extensional viscosity is included with a value close to $\eta_{*}$. By contrast, solutions in the limit $\eta \ll \eta_{*}$ of traditional compaction theory $[6,15]$ significantly underestimate the freeness score and furnish a trend with initial weight that is noticeably nonlinear, except at the lowest solid concentrations.

\section{B. The dynamics of freeness}

For a more complete analysis of the freeness dynamics, we interrogate the model solutions. Figure 11 presents solutions for the parameters of the CSF test with $\eta=0.01 \eta_{*}, \eta_{*}$, and $100 \eta_{*}$. 


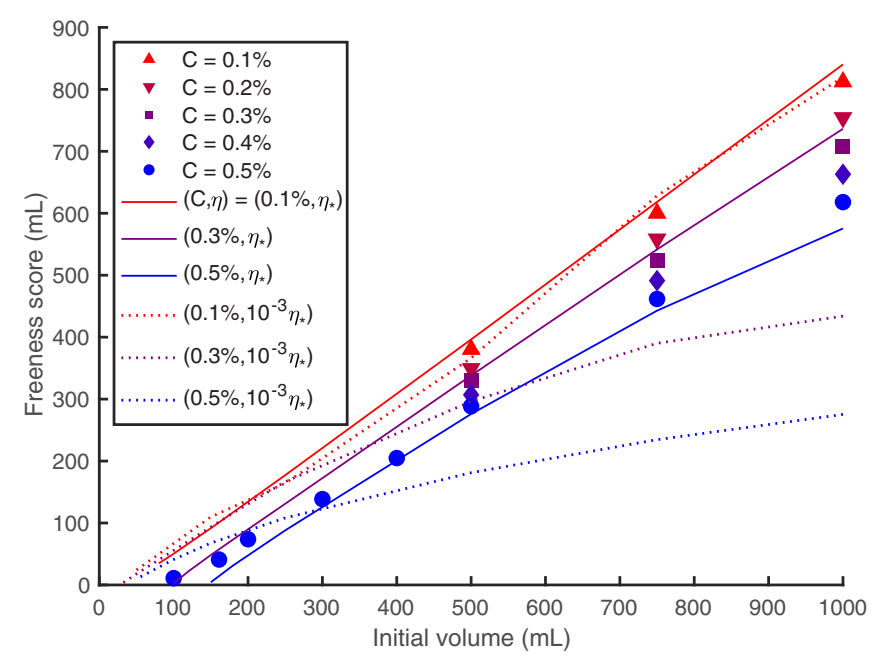

FIG. 10. Freeness score measured experimentally and predicted by the model against initial volume for the initial concentrations $C$ (by mass) indicated. Solid lines show the model results for $\eta=\eta_{*}$, and dotted lines for $\eta=10^{-3} \eta_{*}$.

Shown are the predictions for $h(t)$ and a selection of snapshots of $\phi$ and solid velocity during the period before the discharge from the side channel in the cone switches off. For small solid extensional viscosity [Figs. 11(b) and 11(e)], the bulk of the material falls as an unyielded plug against the screen, rapidly crushing the material there so that the solid stress and hydrostatic pressure come into balance. A boundary layer forms near the screen in which the solid is significantly compacted, and this layer subsequently chokes the falling plug flow because of its low permeability. Consequently, the initial fall of the suspension is rapid, but soon decelerates. At large solid extensional viscosity [Figs. 11(d) and $11(\mathrm{~g})$ ], the rate-dependent stress significantly supports the suspension, reducing the hydrostatic head and the drainage rate; the material remains roughly uniform and the velocity profiles are linear. Extensional viscosities with $\eta=O\left(\eta_{*}\right)$ lead to solutions with characteristics between these two extremes [Figs. 11(c) and 11(f)]. The different dynamics of the three cases is reflected by the resulting freeness scores [which are $490 \mathrm{ml}$ for Figs. 11(b) and 11(e), $736 \mathrm{ml}$ for Figs. 11(c) and 11(f), and $243 \mathrm{ml}$ for Figs. 11(d) and 11(g)].

\section{The limit of zero solid extensional viscosity}

In the limit $\eta \rightarrow 0$ described by the traditional rate-independent models [6,15], the equations for the freeness problem can be simplified owing to the plastic form of the solid stress: When $\phi_{0}<\phi_{g}$, the release of the flow at the base of the container immediatelly causes the bulk of the solid to move downwards with the speed of the top surface and to compact into a much thinner consolidated layer above the screen. If the screen resistance and the contribution of the solid to the weight are relatively small, then the force balance at the base implies that

$$
\mathcal{P}(0, t)=\rho_{f} g h
$$

Thus, at the moment of release, the solid phase must immediately compact at the base to a solid fraction given by $P_{y}(\phi)=\rho_{f} g h_{0}$. Thereafter, however, the overlying weight declines due to the falling height of the suspension, leaving the stress $\mathcal{P}(0, t)$ below the compressive yield stress $P_{y}[\phi(0, t)]$ and the solid over-consolidated there. Immediately above, the downward flow continues to push solid into the compacted layer, thickening it and forcing the solid to consolidate locally up to a yield stress $P_{y}(\phi)$ that balances the instantaneous overlying weight. In other words, an 

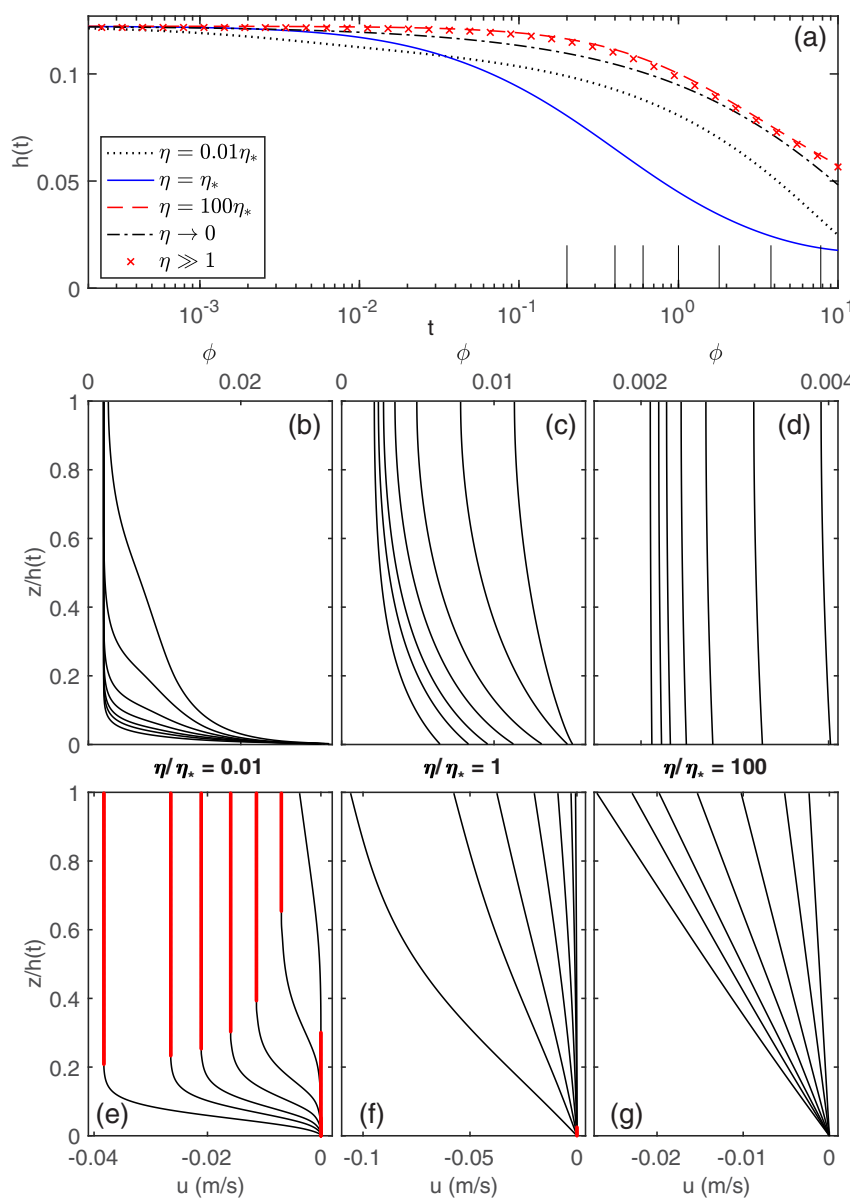

FIG. 11. Model solutions for the freeness test of NBSK for $\eta=0.01 \eta_{*}, \eta_{*}$, and $100 \eta_{*}$. Panel (a) plots the free surface position $h(t)$ against time. Also shown are the asymptotic predictions for $\eta \ll 1$ (Sec. VII B 1) and $\eta \gg 1$ (from Eq. (38) with $\eta=100 \eta_{*}$ ). Panels (b)-(d) and (e)-(g) show snapshots of $\phi$ and $u$ at the times indicated in (a) by thin vertical lines. Thick red lines in (e-g) show unyielded zones, defined for our regularized constitutive law by the condition $|\mathcal{P}|<P_{y}(\phi)$.

upward-migrating compaction front forms at $z=Y(t)$, where

$$
\mathcal{P}(Y, t)=P_{y}(\phi(Y, t))=\rho_{f} g[h(t)-Y(t)]
$$

Above the front, the bulk of the solid still moves down with $\phi=\phi_{0}$, whereas within the compacted layer, the $\phi$-distribution is a frozen record of the evolving yield condition. For $\phi_{0}>\phi_{g}$, this simple structure is complicated by the fact that the solid is gelled above the compaction front and the sharp jump above $z=Y(t)$ begins to diffuse upwards into the bulk of the falling layer. Provided the jump remains sharp, we may approximate it as a discontinuity at the compaction front and apply the mass conservation constraint,

$$
\dot{Y}=\frac{\phi_{0} \dot{h}}{\phi_{0}-\phi(Y, t)},
$$


given that $\phi_{0} \dot{h}$ is the flux into the front from above, and the unyield layer below is stationary. Last, Darcy's law integrated across the unyielded layer implies that

$$
p(Y, t) \equiv \rho_{f} g(h-Y)-P_{y}\left(\phi_{0}\right)=-\mu \dot{h} \int_{0}^{Y} \frac{d z}{k(\phi)}-\rho_{f} g Y
$$

(since $p(0, t)=0$ ), where we have used the hydrostatic pressure $p=\rho_{f} g(h-z)-P_{y}\left(\phi_{0}\right)$ in $Y<$ $z<h$, which includes the contribution of the capillary pressure at the top if $\phi_{0}>\phi_{g}$. Equations (35) and (36), along with Eq. (34), constitute two coupled ODEs for the yield surface and suspension height; the solution for the conditions of the CSF is included in Fig. 11(a). Both the compressive yield stress and permeability feature in this reduced model: $P_{y}(\phi)$ determines the solid distribution at the bottom via force balance, whereas the permeability controls the water flow across the solidified cake above the screen. The main limitation of the approximation in Eqs. (35) and (36) is the diffusive spread of the jump in $\phi$ above the compaction front.

\section{The limit of large solid extensional viscosity}

In the opposite limit of large solid extensional viscosity, the solid becomes almost uniform throughout the compacting column. This implies that $\phi=\phi_{0} h_{0} / h(t)$ and $u=z \dot{h} / h$. The bottom boundary condition now demands that

$$
\dot{h} \sim-\frac{\rho_{f} g}{\Lambda} h^{2} \equiv-\frac{\rho_{f} g}{\eta \phi_{0}^{2} h_{0}^{2}} h^{4},
$$

if the compressive yield stress, screen resistance $\rho_{f} c \dot{h}^{2}$, and solid buoyancy $\phi_{0} h_{0}\left(\rho_{s}-\rho_{f}\right) g$ are all small in comparison to the bulk viscous stress. Thus,

$$
h \sim h_{0}\left(1+\frac{3 \rho_{f} g h_{0}}{\eta \phi_{0}^{2}} t\right)^{-1 / 3}
$$

which is also plotted in Fig. 11(a) for $\eta=100 \eta_{*}$.

In this limit, the freeness score is therefore completely determined by the solid extensional viscosity; the permeability and compressive yield stress play no role. To understand how those additional effects come into play, we estimate the corrections to Eq. (37) as follows: from Eq. (15) we observe that the permeability enters when the Darcy drag is no longer negligible in comparison to the rate-dependent stress, implying

$$
k \frac{\partial}{\partial z}\left(\frac{\Lambda}{\mu} \frac{\partial u}{\partial z}\right) \sim \dot{h}-u \sim\left(1-\frac{z}{h}\right) \dot{h}
$$

The bulk viscous stress at the bottom is then

$$
\Lambda \frac{\partial u}{\partial z}(0, t) \sim \eta \phi_{0}^{2} h_{0}^{2} \frac{\dot{h}}{h^{3}}-\frac{\mu h \dot{h}}{3 k\left(\phi_{0} h_{0} / h\right)} .
$$

Hence, Eq. (37) can be generalized to

$$
P_{y}(\phi)-\eta \phi^{2} \frac{\dot{h}}{h}+\frac{\mu h \dot{h}}{3 k(\phi)}+\rho_{f} c \dot{h}^{2}=\rho_{f} g h+\left(\rho_{s}-\rho_{f}\right) g \phi_{0} h_{0},
$$

with $\phi \sim \phi_{0} h_{0} / h$. Equation (41) can be attacked with dimensional analysis to gauge when effects other than the solid extensional viscosity come into play. In fact, Eq. (41) offers a convenient setting in which to assess parameter sensitivity more generally, as we detail next.

\section{Parameter sensitivity}

To understand in more detail how the freeness score relates to the underlying material behavior of a fibrous suspension, we vary parameters in the model solutions and perform a dimensional 

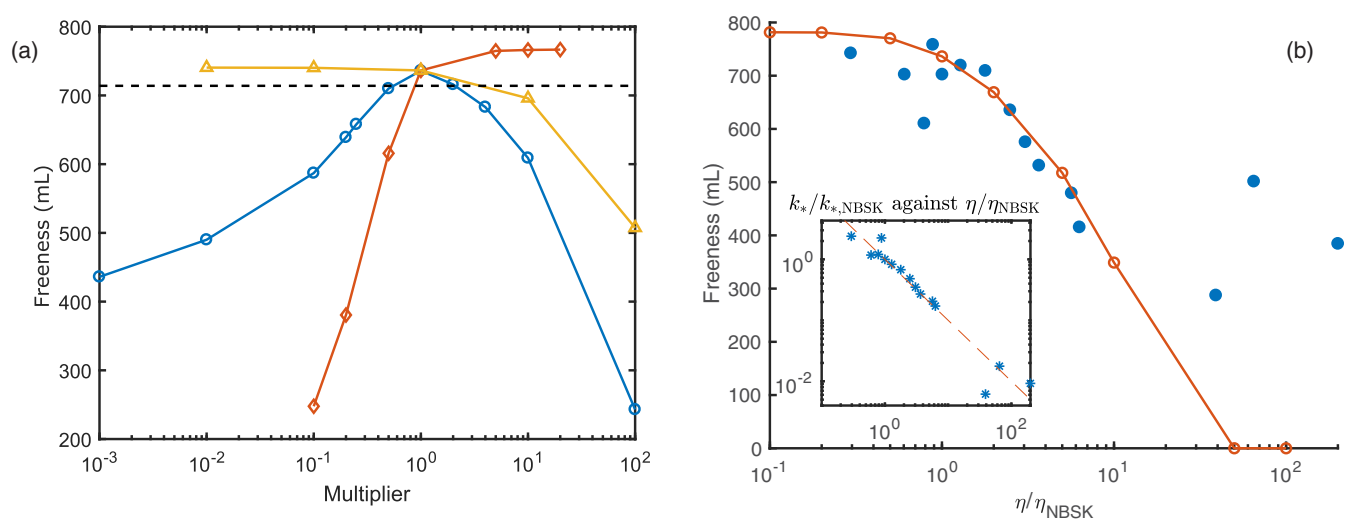

FIG. 12. (a) Freeness score against constant multiplier of $\eta$ (blue circles), $k(\phi)$ (red diamonds) and $P_{y}(\phi)$ (yellow triangles). The experimental freeness of NBSK pulp $(714 \mathrm{ml})$ is shown by dashed line. (b) Freeness score against solid extensional viscosity for the pulp library (filled circles) along with the model predictions for NBSK, varying $\eta$ while keeping the product $\eta k(\phi)$ constant (solid line connecting open circles). The inset shows the inverse correlation between $\eta$ and a characteristic permeability $k_{*}=k(0.1)$.

analysis based on the simplifications afforded by the $\eta \gg 1$ limit exposed above: first, by varying the resistance parameter in the computations, we find that the screen resistance is important in limiting fall speeds at early times. Thus, the initial velocity scale is provided by balancing the screen resistance against the hydrostatic head in Eq. (41), giving

$$
|\dot{h}| \sim \sqrt{\frac{g h_{0}}{c}} \sim 0.07 \mathrm{~m} / \mathrm{s} .
$$

The rate-dependent stress, of order $\eta \phi^{2} \dot{h} / h \sim \eta \phi^{3} \dot{h} /\left(\phi_{0} h_{0}\right)$, only enters in the main balance of Eq. (41) when the compaction at the bottom becomes sufficiently high, which suggests a typical solid fraction there:

$$
\phi(0, t)=\phi_{B} \sim\left(\frac{\rho_{f} g h_{0}^{2} \phi_{0}^{2}}{\eta \dot{h}}\right)^{1 / 4} \sim 5 \times 10^{-3} .
$$

Both estimates in Eqs. (42) and (43) compare well with the model solutions in Figs. 11(c) and 11(f).

Given these estimates, we now gauge the importance of the remaining terms in Eq. (41). From our calibrations of the compressive yield stress, and with $\eta=\eta_{*}$, we find that $\phi_{B} P_{y}\left(\phi_{B}\right) /\left(\rho_{f} g \phi_{0} h_{0}\right) \sim$ 0.03 [since $P_{y}\left(\phi_{B}\right) \sim 10 \mathrm{~Pa}$ ]. Thus, the compressive yield stress is likely to be a relatively small contributor to the freeness score unless it is made larger by a factor of order ten or more. However, the relative size of the term originating from the Darcy drag is $\mu \sqrt{\phi_{0} h_{0} / g c} /\left[3 \rho_{f} k\left(\phi_{B}\right) \sqrt{\phi_{B}}\right] \sim 0.7$ (using $k\left(\phi_{B}\right) \sim 2 \times 10^{-9} \mathrm{~m}^{2}$ ). The permeability is therefore expected to affect the freeness score except when made larger by a factor of ten or so. Last, the overlying solid weight $\left(\rho_{s}-\rho_{f}\right) g \phi_{0} h_{0}$ is negligible in view of the relatively small initial solid fraction.

Since the screen resistance is fixed, these scalings suggest that the solid extensional viscosity and permeability have the most immediate effect on the freeness score, whereas a material requires substantially larger values of $P_{y}$ for the compressive yield stress to become important. These predictions are confirmed in Fig. 12(a), which displays freeness scores obtained from model solutions in which relevant parameters are varied.

More specifically, when we vary $\eta$, holding all the other parameters fixed, we see that the freeness score does indeed depend sensitively on the solid extensional viscosity. Interestingly, the freeness is maximized for $\eta=O\left(\eta_{*}\right)$, and the observed values in the actual freeness tester lie close to the maximum. Evidently, freeness is effectively reduced by the choking of the drainage rate by excessive 
compaction at low solid extensional viscosity, or the viscous support of the suspension at high values of $\eta$. Notably, neither reduction is compatible with the observed freeness score.

Likewise, Fig. 12(a) also highlights how reducing the permeability by a constant factor has a dramatic effect on the freeness score, an effect we attribute to the elevated Darcy drag enhancing the compaction of the solid above the screen. By contrast, increasing the permeability by a constant factor has little effect on freeness, because the Darcy drag is then made unimportant, precisely as anticipated by the scaling analysis. Similarly, changing the compressive yield stress by a constant factor has no effect on the predicted freeness except when $P_{y}(\phi)$ is increased by more than a factor of ten, to enable the solid yield stress to contribute to the support of the hydrostatic load above the screen.

\section{More pulp}

From the preceding discussion, we conclude that the model is able to reproduce the freeness score of NBSK. A rate-dependent stress is certainly needed, and the material parameters that most strongly affect the freeness score are the solid extensional viscosity and the permeability of the pulp matrix. Moreover, the best-fitting choice of the solid extensional viscosity parameter is consistent with that found in pressure filtration tests [16].

To investigate the robustness of these conclusions we have also tested a number of other cellulose suspensions. These include further NBSK mixtures with different chemical additives and other pulps consisting of hardwood fibers or a mixture of hardwood and softwood. Given that the preceding results suggest that the detailed form of the constitutive functions nearer the gel point are not key, we use calibrations of $P_{y}(\phi), k(\phi)$, and $\eta$ from pressure filtration studies [27]. The full set of pulps display a range of material parameters and freeness scores. Notably, however, the permeabilities measured for the pulps also have a remarkable correlation with the solid extensional viscosity (although their compressive yield stresses are similar, varying by at most a factor of two). In particular, given a characteristic measure $k_{*}$ of the permeability at a representative solid fraction, we find that the combination $\eta k_{*}$ is roughly constant over the "library" [see the inset of Fig. 12(b)]. Thus, to compare model predictions with the pulp library, we conduct more computations in which both the solid extensional viscosity and permeability were varied, keeping $\eta k(\phi)$ fixed. As shown in Fig. 12(b), we find a similar trend for the freeness score with $\eta$, although none of the pulps tested have substantially lower values of the bulk viscosity.

\section{DISCUSSION}

In this study we have explored a two-phase model for the flow-induced compaction of a fibrous porous medium over increasingly rapid rates of compaction. Sedimentation and flow-through tests were used to calibrate the permeability and compressive yield stress of the model. The calibrations were bridged to previous fits for higher solid fraction from pressure filtration studies, and they provide a useful characterization of a particular cellulose fiber (NBSK) over almost the entire range of relevant solid fractions. By studying the time-dependence of both these and further drainage tests, we demonstrated that the model was capable of reproducing the experiments beyond the steady state dynamics. Importantly, the model performed significantly better when generalized to incorporate the same bulk solid viscosity used in the previous pressure filtration studies.

We then explored the dynamics of the Canadian Standard Freeness test, whose "freeness score" measures the rapidity of water drainage from a standardized sample of pulp. The model reproduced the observed freeness score for NBSK, both under standard test conditions and over a wider range of initial solid concentrations and suspension volumes. By interrogating the model, we found that the most important material properties for the freeness score are the solid extensional viscosity and the permeability, with the compressive yield stress playing only a minor role. Thus, given that the test is a readily available industrial-standard device, our analysis opens up the possibility of utilising the freeness score more widely for the rheological characterisation of two-phase materials undergoing rapid deformation. 
Nevertheless, because we have focused on a particular experimental material, it is unclear how significant rate-dependent solid stresses might be for different dispersions. In fact, our results for cellulose fibers run somewhat counter to intuition: as remarked by Buscall and White [6], although solid viscous stresses are to be expected in two-phase media, they are normally ignored because dimensional analysis suggests the viscosity to be relatively small. The core of the argument is that the rate-dependent solid stress originates from the viscous flow of the solvent around the solid particles during compaction. Thus, in the low- $\phi$ limit, the solid viscosity scales with that of the solvent, as also predicted by general two-phase flow theory [14]. For our fiber suspension, this suggests that $\Lambda \sim \mu=10^{-3} \mathrm{~Pa}$ s. The bulk viscosity adopted in our model, $\Lambda=\eta \phi^{2}$, is four orders of magnitude larger with the calibrated value for $\eta$ and $\phi=O\left(10^{-3}\right)$. This anomaly mirrors two other curious results: the compressive yield stress depends linearly on $\phi-\phi_{g}$ near the gel point, whereas a stronger dependence is found for other suspensions (Appendix A 2 a), and pulp permeability is unusually low in comparison to other fibers (see Appendix A 2 b).

A rationalization of all these observations requires a micromechanical model of the cellulose fiber suspension, which is beyond our current scope. Nevertheless, we speculate that the unexpected material behavior originates from the structure of the hollow, deformable fibers themselves. The linear increase in the solid stress with $\phi-\phi_{g}$, for example, might arise through elastic bending of individual fibers, either facilitating or preceding a plastic rearrangement of the bulk network. Similarly, the abnormally low permeability may arise because the effective solid fraction is misidentified in the suspension: at low concentrations, the fluid inside the hollow fibers is unable to leak out, implying that the fluid interior should be counted as part of the solid [30]. Finally, because the fibers must be in mechanical contact in the gelled state, it is possible that the relatively high solid viscosity originates not from the larger-scale viscous flow around fibers, but from flow within the much narrower regions where the fibers are in sliding contact. The reduced scale of those regions could, in principle, enhance the viscous dissipation, and therefore the solid viscosity.

Overall, cellulose fiber suspensions constitute an interesting two-phase material with somewhat poorly understood microstructural properties, despite widespread usage. Our efforts here have highlighted the macroscopic rheology that this microstructure must dictate. Whether many other materials share similar properties remains to be seen, although our speculations about the microstructure dynamics are not particularly specific to cellulose.

\section{ACKNOWLEDGMENTS}

We thank Valmet Corp. and the Natural Sciences and Engineering Research Council through the CRD program for financial support.

\section{APPENDIX A: EXTENDED EMPIRICAL FITS}

\section{Extension to higher solid fraction: A withdrawal experiment}

The fits for $P_{y}(\phi)$ and $k(\phi)$ span a relatively low range of solid fractions near $\phi_{g}(\phi \lesssim 0.006)$. By contrast, the fits of these functions from pressure filtration tests [16] cover rather higher solid concentrations $(0.05 \lesssim \phi)$. The order of magnitude gap between these ranges provides a potential source of error when bridging between the two fits via interpolation formulas of the sort outlined below. To shore up the interpolation of the compressive yield stress, we therefore conducted another simple experiment in the flow-through arrangement, designed to extract a cruder estimate of $P_{y}(\phi)$ over a wider range of $\phi$.

More specifically, for an equilibrated test in which the solid had been compressed at the maximum pump rate, we removed the return pipe of the pump from above the pulp chamber and instead withdrew water from the back tank at the same fixed rate. Although the pump rate was at maximum, this withdrawal experiment is still relatively slow, taking about an hour to remove the 121 of water from the two tanks. This leads us to assume that the solid adjusts quasistatically during the withdrawal, allowing us to estimate the compressive yield stress as outlined below. 

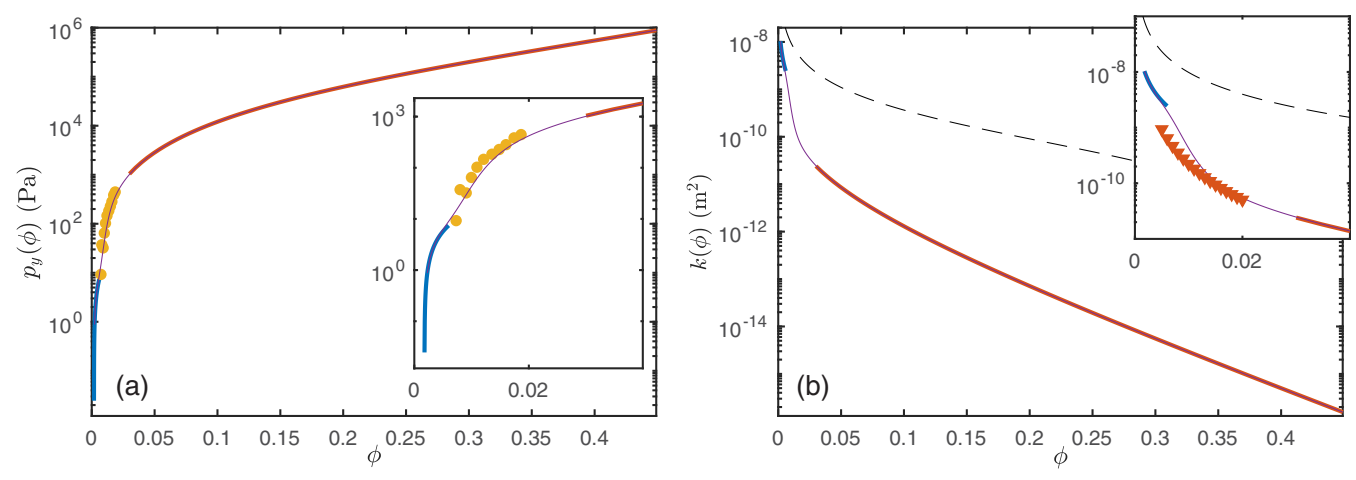

FIG. 13. Plots of the extended fits to the (a) $P_{y}(\phi)$ and (b) $k(\phi)$ functions. In each case, the thicker blue and red lines show the fits from our calibration experiments (Secs. IV and V) and from pressure filtration studies [16], respectively. In panel (a), the dots show the cruder estimates from Appendix A 1. The thinner (purple) lines shows the interpolations of Appendices A 2 a and A 2 b. In panel (b) the fitted permeability for suspensions of rigid fibers of Ref. [20] is plotted with a dashed line, and estimates of an effective bulk permeability obtained via a Pulmac tester are shown with red triangles.

To begin with, the slowly descending top surface of the water remains above the solid. In this state, the draining of the fluid from the arrangement is not expected to affect the pulp, and no changes in the solid were observed during this phase of the experiment. Once the water level in the pulp chamber meets the top of the compacted solid layer, however, capillary stresses prevent drainage through that matrix. The top surface of the suspension then begins to fall less quickly than in the reservoir connected to it because the solid matrix supports a hydrostatic pressure drop across the two. If $2 U_{w}$ denotes the flux at which water is withdrawn, per unit cross sectional area of the two tanks, then

$$
h+h_{b}=2\left(h_{0}-U_{w} t\right)
$$

where $h_{b}$ is the water level in the back reservoir.

At this stage, the pulp is also relatively compacted, with an elevated solid stress countering the capillary pressure that is exerted at $z=h$ to maintain the coincidence of the top surfaces of the water and pulp. This leads us to suppose that the solid becomes relatively uniform, $\phi \approx \phi(t) \approx \phi_{0} h_{0} / h$, in which case $u \approx \dot{h} z / h$. Ignoring the rate-dependent stress in view of the relatively slow withdrawal, Eq. (4) then reduces to

$$
\frac{\partial}{\partial z}\left[P_{y}(\phi)+p\right]=-\rho_{f} g-\phi\left(\rho_{s}-\rho_{f}\right) g .
$$

Given that $P_{y}(\phi)+p=0$ at $z=h, p(0, t)=\rho_{f} g h_{b}$, and $\phi\left(\rho_{s}-\rho_{f}\right) \ll \rho_{f}$, the integral of Eq. (A2) now implies

$$
P_{y}\left(\frac{\phi_{0} h_{0}}{h}\right) \approx 2 \rho_{f} g\left(h-h_{0}+U_{w} t\right)
$$

This allows us to extend the compressive yield stress function to higher $\phi$ using the observed $h(t)$ from the withdrawal experiment, as shown in Fig. 13(a).

\section{Interpolations}

The explicit interpolation formulas for the permeability and compressive yield stress functions are provided below; Fig. 13 illustrates how they bridge between the fits for the experiments of Sec. III and the pressure filtration studies of Ref. [16]. 


\section{a. Compressive yield stress}

Our fit for the compressive yield stress is

$$
P_{y}=\exp \left\{\log \left[m\left(\phi-\phi_{g}\right)\right] S+[1-S] \log \left[\frac{B \phi^{j}}{(1-\phi)^{l}}\right]\right\},
$$

where the interpolant is

$$
S(\phi)=\frac{1}{2}\left[1-\tanh \Upsilon\left(1-\frac{\log \phi}{\log \Phi}\right)\right],
$$

and the parameters are $\Phi=0.01$ and $\Upsilon=10$. The sedimentation tests give $m=1756 \mathrm{~Pa}$ and $\phi_{g}=$ 0.00178 . Pressure filtration studies provide the fitting parameters $B=6 \times 10^{5} \mathrm{~Pa}, j=1.84$ and $l=3.12$.

Note that the fit of $P_{y}(\phi)$ implies an unphysical maximum packing of unity. Part of the reason for this is that the pressure filtration studies do not access sufficiently high $\phi$ that one can reliably approach this limit. The linear behavior near the gel point is also somewhat surprising, with extrapolations from higher solid fraction suggesting the low- $\phi$ limit $P_{y}(\phi) \sim \phi^{2}$ for fibrous suspensions [16,31], and the stronger dependence $P_{y} \propto\left(\phi-\phi_{g}\right)^{n}$, with $n$ between 2 and 4, for other flocculated dispersions [6].

\section{b. Permeability}

For the permeability we use

$$
k=\exp \left\{\log \left[\frac{A}{\phi} \log \left(\alpha \phi^{-1}\right)\right] S+[1-S] \log \left[\frac{\hat{A}}{\phi} e^{-d \phi} \log \phi^{-1}\right]\right\} .
$$

The flow-assisted compaction tests indicate that $A=3 \times 10^{-12} \mathrm{~m}^{2}$ and $\alpha=0.84$, while the pressure filtration studies give $\hat{A}=3.6 \times 10^{-13} \mathrm{~m}^{2}$ and $d=18.52$.

The fit Eq. (A6) exploits the interpolant function $S(\phi)$ of the compressive yield stress function in Eq. (A5) because the results of the withdrawal experiment do not constrain $k(\phi)$. However, the interpolation is supported by an independent set of permeability measurements of the same pulp using a commercial device (a Pulmac tester), which is also included in Fig. 13(b). This device places the pulp under a given compression and a given pore pressure drop to measure the mean permeability of a sample. Differential compaction at low $\phi$ implies that these measurements become inaccurate, and too low in comparison to the actual permeability for $\phi \rightarrow \phi_{g}$.

The pulp permeability is anomalously low in comparison to what is expected for other fibrous porous media. This can be judged by the comparison between the data in Fig. 13(b) and a fit provided by Jackson and James [20], which is also included (using the fiber radius of our pulp). The measured permeabilities are orders of magnitude below the Jackson and James fit over the full range of solid fraction, particularly at higher $\phi$, in agreement with previous studies (see Refs. [16,32]).

Another perspective on this anomaly is provided by the fit of the constant $A$, which in the dilute limit should equal $a^{2}$, where $a$ is the typical radius of a pulp fiber, times a constant between 0.125 and 0.25 dependent on fiber orientation [21]. For NBSK, the typical fiber radius is about $15 \mu \mathrm{m}$, implying that $A$ should be of order $(3-6) \times 10^{-11} \mathrm{~m}^{2}$, which is an order of magnitude larger than the fitted value quoted in Sec. V. This may arise because the dilute theory assumes that the fibers are solid and straight rigid rods, whereas in reality the cellulose is hollow and deformable. Moreover, the conduits in the fiber walls through which the water inside may leak out are small, suggesting that at low solid fractions, it may be more appropriate to consider the fluid contents of the fibers as part of the solid matrix (cf. Ref. [30]). Thus, the effective solid fraction is higher than expected by a factor corresponding to the volume ratio of the fiber to its interior, which is about five for NBSK [33]. 


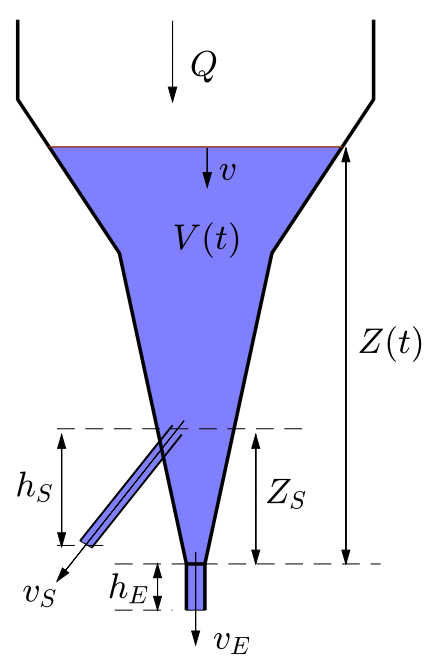

FIG. 14. Sketch of the funnel geometry of the freeness device.

\section{APPENDIX B: FREENESS CONE}

A sketch of the cone in the freeness device is shown in Fig. 14. Water is fed in at the top with a flux $Q(t)$ and fills the main chamber of the freeness device to a depth of $Z(t)$; just below that interface, the instantaneous (downward) flow speed is $v$. At the bottom of the chamber, water enters an exit channel with flow speed $v_{E}$. A side channel with an entrance at a height $Z_{S}=6.6 \mathrm{~cm}$ above the bottom of the chamber removes water with speed $v_{s}$ to register the freeness score.

Taking atmospheric pressure to be zero and assuming that the flow inside the cone is quasisteady, we apply Bernoulli's law to determine the pressures at the bottom of the vessel and the entrance to the side channel,

$$
p_{E}+\frac{1}{2} \rho v_{E}^{2}=\rho g Z+\frac{1}{2} \rho v^{2}
$$

and

$$
p_{S}+\rho g Z_{S}+\frac{1}{2} \rho v_{s}^{2}=\rho g Z+\frac{1}{2} \rho v^{2},
$$

respectively. We also adopt the friction factors $f_{E} \approx 0.14$ and $f_{S} \approx 0.36$ to model the resistance of the two outflow channels (as estimated by circulating water through the cone at various constant flow rates), so that

$$
p_{E}=f_{E} \rho v_{E}^{2}-\rho g h_{E} \quad \text { and } \quad p_{S}=f_{S} \rho v_{S}^{2}-\rho g h_{S},
$$

where $h_{E}=1 \mathrm{~cm}$ and $h_{S}=7 \mathrm{~cm}$ denote the vertical lengths of the exit and side channels. Thus,

$$
v_{E}=\sqrt{\frac{v^{2}+2 g\left(Z+h_{E}\right)}{\left(1+2 f_{E}\right)}}
$$

and

$$
v_{s}=\sqrt{\frac{v^{2}+2 g\left(Z-Z_{s}+h_{s}\right)}{\left(1+2 f_{s}\right)}}
$$

provided $Z>Z_{S}$ the side-channel discharge switches off if $Z<Z_{S}$.

Mass conservation within the vessel demands that

$$
\dot{V}=A \dot{Z}=Q-A_{E} v_{E}-A_{S} v_{S},
$$


where $A(Z), A_{E}=0.086 \mathrm{~cm}^{2}$ and $A_{S}=1.29 \mathrm{~cm}^{2}$ are the horizontal cross-sectional areas at the top free surface within the cone and of the exit and side channels, respectively, and the volume of water in the main compartment $V$ can be related to $Z$ through an empirical relation accounting for the geometry; a simple fit suggests that $V(Z)=442 Z^{9}-0.639 Z^{6}+0.060 Z^{3}+1.7 \times 10^{-5} \mathrm{~m}^{3}$ (measuring $Z$ in meters).

We simplify further by observing that in the freeness test the main compartment fills up to a depth of order tens of centimeters in a few seconds. Thus $\dot{Z}=O(0.1) \mathrm{m} / \mathrm{s}$. By contrast, $\left(v_{E}, v_{S}\right) \sim$ $\sqrt{g Z}=O(1) \mathrm{m} / \mathrm{s}$. This disparity implies that the flow within the cone is quasisteady, and suggests that we reduce Eq. (B6) to

$$
\dot{Z}=\frac{Q}{A}-\frac{A_{E}}{A} \sqrt{\frac{2 g\left(Z+h_{E}\right)}{1+2 f_{E}}}-\Theta \frac{A_{S}}{A} \sqrt{\frac{2 g\left(Z-Z_{S}+h_{S}\right)}{1+2 f_{S}}},
$$

where $\Theta=1$ if $Z>Z_{s}$ and $\Theta=0$ otherwise. We solve Eq. (B7), starting from $Z(0)=0$ and given the input flux $Q(t)$, up until the height $Z(t)$ falls back below $Z_{s}$. The integral of the discharge through the side channel provides the freeness score.

As a check of the applicability of the model, we perform two tests: first, we record the freeness scores (i.e., the total discharge through the side channel) when draining a fixed amount of water from the cone. For 11 of water in the cone, the model predicts a score of $902 \mathrm{ml}$, in comparison to a measurement of $899 \mathrm{ml}$; for $500 \mathrm{ml}$ of water, the theoretical score is $440 \mathrm{ml}$, whereas we observe $436 \mathrm{ml}$. Second, conducting the freeness test itself with pure water (and using the calibrated value for the screen friction factor quoted earlier, $c=180$ ) we calculate a score of $903 \mathrm{ml}$, in comparison to the operating range of $880-890 \mathrm{ml}$ stated in the CSF documentation.

Earlier models [22-24] adopt a rather simpler description of the cone, taking the flux through the bottom to be a fixed discharge (i.e., $A_{E} v_{E} \approx 8.833 \mathrm{ml} / \mathrm{s}$ ) and assuming the main compartment fills instantaneously. This approximation allows one to relate the influx $Q$ directly to the side-channel discharge $A_{s} v_{s}$. If we introduce the same assumption into Eq. (B6), then we find that the freeness score is higher by roughly 20 points.

[1] K. S. Coe and G. H. Clevenger, Methods for determining the capacities of slime-settling tanks, Trans. AIME 55, 203 (1916).

[2] B. F. Ruth, Studies in filtration III. Derivation of general filtration equation, Ind. Eng. Chem. 27, 708 (1935).

[3] G. J. Kynch, A theory of sedimentation, Trans. Faraday Soc. 48, 166 (1952).

[4] K. Terzaghi, Theoretical Soil Mechanics (John Wiley \& Sons, Inc., New York, 1943).

[5] M. A. Biot, General theory of three-dimensional consolidation, J. Appl. Phys. 12, 155 (1941).

[6] R. Buscall and L. R. White, The consolidation of concentrated suspensions. Part 1. The theory of sedimentation, J. Chem. Soc., Faraday Trans. 1 83, 873 (1987).

[7] D. M. Audet and A. C. Fowler, A mathematical model for compaction in sedimentary basins, Geophys. J. Int. 110, 577 (1992).

[8] F. Concha and R. Bürger, A century of research in sedimentation and thickening, KONA Powder Part. J. 20, 38 (2002).

[9] R. G. De Kretser, D. V. Boger, and P. J. Scales, Compressive rheology: An overview, Rheol. Rev., 125 (2003).

[10] J. Olivier, J. Vaxelaire, and E. Vorobiev, Modelling of cake filtration: An overview, Sep. Sci. Technol. 42, 1667 (2007).

[11] A. D. Stickland and R. Buscall, Whither compressional rheology? J. Non-Newtonian Fluid Mech. 157, 151 (2009). 
[12] J. F. Morris and F. Boulay, Curvilinear flows of noncolloidal suspensions: The role of normal stresses, J. Rheol. 43, 1213 (1999).

[13] M. Spiegelman, Flow in deformable porous media. Part 1 Simple analysis, J. Fluid Mech. 247, 17 (1993).

[14] D. A. Drew and S. L. Passman, Theory of Multicomponent Fluids (Springer Science and Business Media, Berlin, 2006), Vol. 135.

[15] K. A. Landman and L. R. White, Solid/liquid separation of flocculated suspensions, Adv. Colloid Interface Sci. 51, 175 (1994).

[16] D. R. Hewitt, D. T. Paterson, N. J. Balmforth, and D. M. Martinez, Dewatering of fiber suspensions by pressure filtration, Phys. Fluids 28, 063304 (2016).

[17] D. R. Hewitt, J. S. Nijjer, M. G. Worster, and J. A. Neufeld, Flow-induced compaction of a deformable medium, Phys. Rev. E 93, 023116 (2016).

[18] C. W. MacMinn, E. R. Dufresne, and J. S. Wettlaufer, Fluid-Driven Deformation of a Soft Granular Material, Phys. Rev. X 5, 011020 (2015).

[19] M. Kvick, D. M. Martinez, D. R. Hewitt, and N. J. Balmforth, Imbibition with swelling: Capillary rise in thin deformable porous media, Phys. Rev. Fluid 2, 074001 (2017).

[20] G. W. Jackson and D. F. James, The permeability of fibrous porous media, Canadian J. Chem. Eng. 64, 364 (1986).

[21] J. J. L. Higdon and G. D. Ford, Permeability of three-dimensional models of fibrous porous media, J. Fluid Mech. 308, 341 (1996).

[22] F. El-Hosseiny and J. F. Yan, Analysis of Canadian standard freeness. I. Theoretical considerations, Pulp Pap.-Canada 81, 61 (1980).

[23] P. C. Swodzinski and M. R. Doshi, Mathematical models of Canadian standard freeness (CSF) and Schopper-Riegler freeness (SR), Proc. Int. Conf. Process Mater. Qual. Eval., 253 (1986).

[24] P. Kumar, H. L. Wei, B. V. Ramarao, and M. Doshi, A model for freeness measurement of papermaking suspensions, Chem. Eng. Commun. 152, 287 (1996).

[25] E. W. K. Young, The permeability and compressibility of semi-dilute pulp fiber suspensions, MASc thesis, Department of Mechanical Engineering, University of British Columbia, 2001.

[26] I. A. Frigaard and C. Nouar, On the usage of viscosity regularization methods for visco-plastic fluid flow computation, J. Non-Newtonian Fluid Mech. 127, 1 (2005).

[27] D. T. Paterson, Understanding rapid dewatering of cellulose fiber suspensions, MASc thesis, Department of Mechanical Engineering, University of British Columbia, 2016.

[28] F. M. Auzerais, R. Jackson, and W. B. Russel, The resolution of shocks and the effects of compressible sediments in transient settling, J. Fluid Mech. 195, 437 (1988).

[29] F. M. Auzerais, R. Jackson, W. B. Russel, and W. F. Murphy, The transient settling of stable and flocculated dispersions, J. Fluid Mech. 221, 613 (1990).

[30] A. A. Robertson and S. G. Mason, Specific surface of cellulose fibers by the liquid permeability method, Pulp Paper Mag. Can. 50, 103 (1949).

[31] F. Tapia, S. Shaikh, J. E. Butler, O. Pouliquen, and E. Guazzelli, Rheology of concentrated suspensions of non-colloidal rigid fibers, J. Fluid Mech. 827, R5 (2017).

[32] J. D. Lindsay and P. Brady, Studies of anisotropic permeability with applications to water removal in fibrous webs part 1. Experimental methods, sheet anisotropy, and relationships to freeness, Institute of Paper Science and Technology, Atlanta, GA (1993).

[33] N. J. McIntosh, Y. Sharma, A. B. Phillion, J. A. Olson, and D. M. Martinez, Estimating the transverse dimensions of cellulose fibers in wood and paper using 2D and 3D microscopy techniques, Cellulose 26, 2099 (2019). 